\title{
VALUACIÓN DE UN CONTRATO DE CONCESIÓN PETROLERA: ANÁLISIS DEL RIESGO, SIMULACIÓN Y OPCIONES REALES CON VOLATILIDAD CAMBIANTE
}

VALUATION OF AN OIL LICENSE AGREEMENT: RISK ANALYSIS, SIMULATION AND REAL OPTIONS WITH CHANGING VOLATILITY

Gastón Silverio Milanesi ${ }^{1}$

https:/ / doi.org/10.52292/j.eca.2021.2017

Fecha de recepción: 15/05/2020

Fecha de aceptación: 06/08/2020

\section{Resumen}

El objetivo del trabajo consiste en exponer diferentes maneras de valuar un contrato de concesión con opciones de renovación y abandono secuenciales para el yacimiento Vaca Muerta en Argentina. En tal sentido, se utiliza la valuación por múltiplos, descuento de flujos de fondos y opciones reales con volatilidad cambiante. El análisis se complementa con el uso de planillas de cálculo para estudiar el riesgo del proyecto: sensibilidad, escenarios y simulación. Esta última permite calcular descripciones estadísticas, probabilidades estandarizadas, ratios de ganancias-pérdidas y volatilidad, estimada mediante el enfoque MAD (marketed asset disclaimer). Los resultados obtenidos exponen el alcance y las limitaciones de los modelos empleados. Se concluye a favor del uso de modelos de opciones con volatilidad cambiante, porque permiten la

1 Departamento de Ciencias de la Administración. Universidad Nacional del Sur. E-mail: milanesi@uns.edu.ar. ORCID: https://orcid.org/0000-0003-1759-6448 
valuación de la flexibilidad estratégica, sensibilizando las principales variables y analizando el impacto, en el valor y condiciones contractuales.

Palabras claves: valuación, riesgo, simulación, opciones reales, volatilidad cambiante.

\begin{abstract}
This article aims to explain different ways of valuing an Oil License Agreement with sequential options of abandonment and renewal for the Vaca Muerta field in Argentina. For that purpose, the multiple, discount cash flow and real options with changing volatility valuation is used. To complement the analysis spreadsheets are used to study project risk: sensibility, scenarios and simulation. The latter allows calculating statistic descriptions, standardized odds, profit-loss ratios and volatility, estimated with MAD (marketed asset disclaimer) approach. The results show the scope and limitations of the employed models. It is concluded that the best one is the real options model with changing volatility because it allows strategic flexibility valuation, raising awareness of the main variables and analyzing the impact on value and agreement conditions.
\end{abstract}

Key words: Valuation, Risk, Simulation, Real Options, Changing Volatility.

JEL: G17, G31. 
Valuación de un contrato de concesión petrolera: análisis del riesgo, simulación... https://doi.org/10.52292/j.eca.2021.2017

\section{Introducción}

Un proceso de valuación es una actividad intelectual donde el conocimiento adquirido sobre la unidad de análisis se transforma en parámetros cuantitativos de valor, con el fin de simplificar el proceso de toma de decisiones. En tal sentido, los modelos de valuación son herramientas que permiten sintetizar y explicar un conjunto de datos, variables y relaciones, que conjugadas en la lógica del modelo permiten cuantificar el valor esperado y el desvío sobre la estimación.

En ese sentido el trabajo busca desarrollar y aplicar un modelo de valuación sobre un contrato de concesión de exploración y explotación petrolera, basado en la teoría de opciones reales (Dixit \& Pindyck, 1994; Trigeorgis, 1995; Smith \& Nau, 1995; Trigeorgis, 1997; Amram \& Kulatilaka, 1998; Copeland \& Antikarov, 2003; Smit \& Trigeorgis, 2004; Smith, 2005; Brandao, Dyer \& Hahn, 2005; Milanesi, 2013a, 2013b; Num, 2015; Milanesi, Pesce \& El Alabi, 2016; Milanesi, 2018). Se toma como base un modelo numérico binomial con volatilidad cambiante (Haahtela, 2011; Milanesi, 2014) y simulación MAD (marketed asset disclaimer) para el steady-state de la concesión, incorporando el valor de las opciones contenidas en el pliego.

El clásico modelo de descuento de flujos de fondos (Booth, 2002, 2007) basa su valor sobre la perspectiva del inversor eficientemente diversificado, de allí la necesidad de tasa de descuento ajustada por riesgos sistemáticos propios de una inversión en una firma en marcha. El valor obtenido refleja la pasividad de una tenencia accionaria o parte de capital en una cartera eficientemente diversificada. El enfoque de opciones reales parte de una premisa: cuantificar la flexibilidad estratégica, es decir, los diferentes cursos de acción que adopta la firma. Es por ello que su enfoque se adapta más a la idiosincrasia gerencial. Consideran todo el riesgo del proyecto y valoran la flexibilidad estratégica, prescindiendo del uso de tasas ajustadas por riesgo (Ahn \& Wilmott, 2009; Copeland \& Antikarov, 2003; Milanesi, 2012, 2014; Smith \& Nau, 1995).

El trabajo se estructura de la siguiente manera: la sección 2 expone las ecuaciones del modelo binomial con volatilidad cambiante y estimación el valor de continuidad en el steady-state. Seguidamente, siguiendo la metodología de análisis de casos en administración, se exponen los aspectos descriptivos de la unidad de análisis. La cuarta sección presenta los resultados obtenidos de aplicar múltiplos, descuento de flujos de fondos y análisis del riesgo. Finalmente, se aplica el modelo de opciones reales, su volatilidad cambiante y la valuación de la flexibilidad estratégica, simulando las condiciones económicas y analizando su impacto en el valor. 


\section{Marco teórico: desarrollo de un modelo de valuación de un contrato de concesión con opciones reales y volatilidad cambiante}

Se desarrollan las ecuaciones correspondientes a la estructura del modelo de valuación propuesto. El punto de partida lo constituye el clásico modelo binomial ${ }^{2}$ CRR (Cox, Ross \& Rubinstein, 1979). Sus parámetros son los siguientes:

$$
\begin{gathered}
u=e^{\sigma \sqrt{ } \Delta t} \\
d=e^{-\sigma \sqrt{ } \Delta t} \\
p=\frac{e^{r \Delta t}-d}{u-d}
\end{gathered}
$$

Las ecuaciones precedentes corresponden a las expresiones para estimar el coeficiente de ascenso, descenso y probabilidad neutral al riesgo. La versión general del modelo para $n$ períodos y j ésimos nodos es:

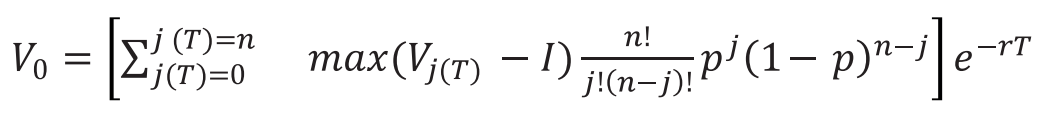

En la anterior expresión, representa el valor terminal de una opción real asimilable a una opción de compra ${ }^{3}, n$ el horizonte de tiempo, $j$ el j-ésimo nodo, $p$ la probabilidad neutral al riesgo, factor de descuento al tipo sin riesgo.

En este caso, siguiendo la propuesta de Haahtela (2011a, 2011b), las probabilidades de transición deben reconfigurarse, asegurando que la rejilla no pierda capacidad de recombinar nodos, y permita trabajar con diferentes niveles de

2 Existen varias formulaciones del modelo binomial, en función a como se definen las restricciones y parámetros, en particular la restricción de recombinación (Chance, 2007; Van der Hoek \& Elliot, 2006; Whaley, 2006).

3 Donde VT(j) representa el valor del subyacente en T, I es la inversión (ejercicio), por ejemplo diferimiento, expansión, incremento de capacidad operativa, inclusive el mismo valor de la empresa para calcular probabilidades de insolvencia (Milanesi, 2016). En el caso de estrategias asimilables a opciones de venta, la expresión queda planteada como maxi(I-Vj(T) ), por ejemplo abandono, transferencia total o parcial, quiebra de la firma. 
volatilidad. Primero se deben estimar las probabilidades de transición para los máximos niveles de volatilidad,

$$
p i_{(\max )}=\frac{e^{r \Delta t}-d_{\max }}{u_{\max }-d_{\max }}
$$

Las probabilidades de transición máximas ( son obtenidas a partir de los coeficientes de ascenso y descenso (ecuaciones 1 y 2 ) estimados con la máxima volatilidad. Seguidamente son calculadas las probabilidades de transición para los períodos de menor volatilidad, obtenidas a partir de proporcionar la volatilidad del período sobre la máxima y ajustando la probabilidad,

$$
p i_{(\sigma)}=p_{u(\max )}\left(\frac{\sigma_{i}}{\sigma_{\max }}\right)^{2}
$$

Se proyecta el comportamiento del subyacente, por ejemplo, el precio del commodity ( estudiado, con la siguiente expresión

$$
P_{t+1}^{i, j}=P_{t}^{i, j} \times u_{\max } ; P_{t}^{i, j} \times d_{\max }
$$

La proyección utiliza los máximos valores correspondientes para los movimientos de ascenso y descenso. Asumiendo perfecta correlación entre precios y costos, y suponiendo que el proceso técnico de producción (unidades) se ajusta a los parámetros proyectados ${ }^{4}$, el flujo de fondos operativos para cada nodo se obtiene mediante las siguientes expresiones:

$$
\begin{gathered}
F F O_{t}^{i, j}=P_{t}^{i, j} \times E B I T \%(1-t) \\
F F O(\text { total })_{t}^{i, j}=F F O_{t}^{i, j} \times B b l(t)
\end{gathered}
$$

4 Caso contrario se debe trabajar con opciones del tipo arco iris con múltiples fuentes de incertidumbre, independientes o correlacionadas, ver Brous (2011), Copeland \& Antikarov (2003), Milanesi (2011). 
Las ecuaciones 8 y 9 permiten calcular el flujo de fondos operativos por unidad y total en cada nodo de la rejilla. Deduciendo la inversión incremental en capital de trabajo, se obtiene el flujo de fondos libres:

$$
F F L_{t}^{i, j}=F F O(\text { total })_{t}^{i, j}-\left(F F O(\text { total })_{t}^{i, j} \times \Delta C T_{t} \%\right)
$$

El valor de la firma en cada nodo se integra por su flujo de fondos libres $\left(F F L_{t}^{i, j}\right.$ ) y el correspondiente valor de la empresa. Al llegar al horizonte (T) el valor de la forma en cada nodo equivale al valor de continuidad (steady-state). Este se obtiene como resultado del producto entre el flujo de fondos libres en cada nodo del horizonte y la anualidad, esta última reflejando la renta hasta el horizonte final pautado ${ }^{5}$ :

$$
V_{T, i, j=} F F L_{T}^{i, j} \times A_{r, T+n}
$$

El proceso recursivo comienza desde el último período, $V_{T-1, i, j=}\left[V_{T, j} \times p_{i(\sigma)}+\right.$ $\left.V_{T, j} \times\left(1-p_{i(\sigma)}\right)\right] e^{-r f}$ hasta el momento $\mathrm{t}=0$, donde desde T-1 se deben incorporar los flujos de fondos:

$$
V_{t, i, j=} F F L_{t}^{i, j}+\left[V_{t+1, j} \times p_{\sigma t}+V_{t+1, j} \times\left(1-p_{\sigma t}\right)\right] e^{-r f}
$$

El planteo de opciones se hace en la ecuación 12,

$$
V_{t, i, j}=\max \left\{F F L_{t}^{i, j}+\left[V_{t+1, j} \times p_{\sigma t}+V_{t+1, j} \times\left(1-p_{\sigma t}\right)\right] ; O R_{1} \ldots . O R_{n}\right\} e^{-r f} \mathrm{Ec}
$$

5 En un empresa en marcha tiende a infinito, por ende el valor terminal en estado de ste$a d y$-state es $\left(\llbracket F F L \rrbracket_{-} t^{\wedge}(i, j)\right) / r$, ya que $A r, \infty=1 / r$. En una concesión con vigencia finita, Ar,$\mathrm{t}=(1+\mathrm{r}) \mathrm{T}+\mathrm{n}-1(1+\mathrm{r}) \mathrm{T}+\mathrm{n} \times \mathrm{r}$. 
Valuación de un contrato de concesión petrolera: análisis del riesgo, simulación...

https://doi.org/10.52292/j.eca.2021.2017

\section{Metodología: análisis de caso concesión de exploración y extracción de petróleo}

La presente sección tiene por objeto presentar los aspectos técnicos y variables económicas correspondientes a la unidad de análisis seleccionada. El estudio de casos en administración es una metodología que profundiza las interrelaciones existentes entre las variables que componen un modelo (Castro Monge, 2010).

La unidad de análisis está dada por un contrato de concesión de exploración y explotación en la zona Vaca Muerta, provincia de Neuquén, República Argentina. Su actividad consiste en la perforación de cuatro pozos horizontales en la zona de Loma Amarilla, de 2500 metros de rama horizontal en un área de $175 \mathrm{~km}^{3}$. Los datos correspondientes a todas las variables corresponden a febrero del año 2020.

La unidad de medida para estimar ingresos es el barril de petróleo tipo Brent. Se estima que la vida útil de este tipo de pozos ronda entre los 20 y 30 años, fijándose para este caso un horizonte de 20 años, o 7300 días $^{6}$. Considerando los datos técnicos correspondientes a reservas promedio en este tipo de pozos, se presenta el cuadro de producción estimado.

Tabla 1. Producción proyectada mensual, anual, acumulada por pozo promedio

\begin{tabular}{c|c|c|c|c|c|c|c}
\hline Año & $\begin{array}{c}\mathrm{m}^{3} \\
\text { mensual }\end{array}$ & $\begin{array}{c}\mathrm{Bbl} \\
\text { mensual }\end{array}$ & $\begin{array}{c}\mathrm{m}^{3} \\
\text { anual }\end{array}$ & $\begin{array}{c}\mathrm{Bbl} \\
\text { anual }\end{array}$ & $\begin{array}{c}\text { Acumulado } \\
\mathrm{m}^{3}\end{array}$ & $\begin{array}{c}\text { Acumulado } \\
\text { Bbl }\end{array}$ & \% reservas \\
\hline 1 & 3500 & 22.015 & 42.000 & 26.4180 & 42.000 & 264.180 & $77,78 \%$ \\
\hline 2 & 2800 & 17.612 & 33.600 & 211.344 & 75.600 & 475.524 & $60,00 \%$ \\
\hline 3 & 1950 & $12.265,5$ & 23.400 & 147.186 & 99.000 & 622.710 & $47,62 \%$ \\
\hline 4 & 1200 & 7548 & 14.400 & 90.576 & 113.400 & 713.286 & $40,00 \%$ \\
\hline 5 & 800 & 5032 & 9600 & 60.384 & 123.000 & 773.670 & $34,92 \%$ \\
\hline 5 a 10 & 550 & 3459,5 & 6600 & 41.514 & 156.000 & 981.240 & $34,92 \%$ \\
\hline 10 a 20 & 275 & 1729,75 & 3300 & 20.757 & 189.000 & 1.188 .810 & $17,46 \%$ \\
\hline
\end{tabular}

Fuente: elaboración propia a partir de datos secundarios ${ }^{7}$.

6 Ver https://www.worldenergytrade.com/index.php/m-news-oil-gas/86-news-produccion/3728-se-alarga-la-vida-de-los-pozos-en-vaca-muerta-hasta-por-30-anos

7 https://www.worldenergytrade.com/index.php/m-news-oil-gas/86-news-produccion/3728-se-alarga-la-vida-de-los-pozos-en-vaca-muerta-hasta-por-30-anos 
La tabla 1 discrimina la producción promedio para este tipo de pozo en mensual, anual y acumulada en metros cúbicos y por barril. La última columna indica el porcentaje de reserva remanente transcurrido el período de tiempo, donde a partir del cuarto año la curva de producción tiende a aplanarse, estabilizándose totalmente a partir del décimo año.

En relación con los costos, se suponen de 33 USD por barril, conforme el detalle de la tabla 2.

Tabla 2. Costos de desarrollo, extracción y regalías por barril

\begin{tabular}{c|c}
\hline A-Desarrollo & \\
\hline Recupero de capital & $\$ 10,50$ \\
\hline B-Lifting cost & $\$ 8,13$ \\
\hline Directos producción & $\$ 4,38$ \\
\hline Indirectos producción & \\
\hline C-Otros & $\$ 10,00$ \\
\hline Regalías e impuestos & $\$ 33,01$ \\
\hline Costo Total por Barril (A+B+C) & \\
\hline
\end{tabular}

Fuente: elaboración propia con base en datos disponibles online ${ }^{8}$.

Cabe destacar que el break-even por barril, en Estados Unidos, actualmente se estima en 20 USD, siendo para este caso un valor entre 30 USD y 40 USD dependiendo básicamente del factor tributario. El valor de la concesión e inversiones requeridas asciende a 60 millones de dólares estadounidenses ${ }^{9}$, estimándose una inversión en capital de trabajo del 1,98 \% promedio sobre ingresos (Non-Cash WC/Sales), para el sector Oil/Gas exploración y producción en

8 https://www.lmneuquen.com/ypf-el-costo-produccion-us-40-barril-n626668, https:/ / www.iprofesional.com/notas/236864-petrleo-ypf-barril-Cuanto-le-sale-a-YPF-extraer-unbarril-de-petroleo

9 Ver Revista Petroquímica: Petróleo, Gas, Química y Energía (https://www.revistapetroquimica. com/vaca-muerta-neuquen-adjudico-dos-nuevas-concesiones-por-us-90-millones/) 
Valuación de un contrato de concesión petrolera: análisis del riesgo, simulación... https://doi.org/10.52292/j.eca.2021.2017

el mercado norteamericano con base en 269 empresas $^{10}$. Uno de los supuestos para la proyección de ingresos y costos es suponer la perfecta correlación positiva entre los componentes, como la perfecta correlación de los costos a la evolución del dólar en el marco del cumplimiento de la teoría de la paridad del poder adquisitivo en Argentina ${ }^{11}$.

Se supone alícuota promedio de impuesto a las ganancias del $30 \%$ sobre la ganancia imponible, concepto no contemplado en el punto c de la tabla 2. El contrato contiene un conjunto de opciones o alternativas estratégicas que otorgan flexibilidad al operador, las cuales se resumen en la tabla 3.

Tabla 3. Opciones estratégicas del contrato

\begin{tabular}{|c|c|c|}
\hline \multicolumn{3}{|c|}{ Condiciones contractuales } \\
\hline Alternativas & $A \tilde{n} o=5$ & Inversión \\
\hline Opción1 & Ceder otro operador & $\$ 35.000 .000,00$ \\
\hline Opción2 & Renovar similar & \$ 16.761 .904 \\
\hline Alternativas & $A \tilde{n} o=10$ & Inversión \\
\hline Opción1 & Ceder otro operador & $\$ 15.000 .000,00$ \\
\hline Opción2 & Renovar similar & \$ 6.285.714 \\
\hline Duración total & 20 años & \\
\hline
\end{tabular}

Fuente: elaboración propia.

Las alternativas son ejercidas en los años 5 y 10, teniendo una duración máxima de 20 años que se corresponde con la capacidad técnica del área. La opción 1 implica ceder la explotación a otro operador, por un valor estimado de 35.000.0000 USD en el año 5 y 15.000.000 USD en el año 10. En su defecto,

10 Damodaran, A.(http://pages.stern.nyu.edu/ adamodar/New_Home_Page/datafile/ wcdata.html).

11 Existe evidencia empírica que Argentina destaca por ajustar perfectamente a la evolución del dólar cumpliéndose la teoría de paridad de poder adquisitivo. Los desequilibrios son coyunturales propios de retrasos cambiarios coyunturales, a raíz de desfasajes entre el tipo de cambio nominal y el real (equilibrio) (Espert \& Vignoli, 2018). 
la opción 2, que implica ejercer el derecho de renovación de la concesión del área, por un valor de 16.761.904 USD para el año 5 (renovándose por 5 años) y 6.285.714 USD para el año 10 hasta el 20. El precio de ejercicio correspondiente a estas opciones fue estimado considerando las reservas técnicas remanentes sobre el valor del pliego original, es decir, a partir del año 5 de 34,92 \% y desde el año 10 al 20 de 17,46 \%, descontando por desgaste a la inversión inicial estimada en un $20 \%$ (año 5) y 40 \% (año 10).

Desde la perspectiva de la valuación financiera y análisis del riesgo, los aspectos relacionados con el proceso de producción y costos asociados, se pueden considerar cuasi endógenos o pseudo controlables ${ }^{12}$. La principal fuente de incertidumbre "de mercado" (Smith \& Nau, 1995) está dada por la evolución del precio del petróleo, siendo el valor del barril Brent la variable a observar. A tal fin se analizó la cotización del Brent durante una ventana de tiempo de 10 años, desde abril del 2010 a marzo $2020^{13}$, tomando horizontes móviles a 1, 5 y 10 años hacia atrás, desde el momento de valoración se tienen los datos estadísticos en relación al precio que se muestran en la tabla 4.

Tabla 4. Volatilidad, precio promedio y variación absoluta del precio Brent a marzo 2020

\begin{tabular}{|c|c|c|c|}
\hline Horizontes & Volatilidad & Precio promedio & Var. Abs. Precio \\
\hline 1 & $49,42 \%$ & $\$ 63,23$ & $\$ 50,22$ \\
\hline 5 & $47,76 \%$ & $\$ 58,42$ & $\$ 27,90$ \\
\hline 10 & $38,68 \%$ & $\$ 79,92$ & $\$ 30,91$ \\
\hline
\end{tabular}

Fuente: elaboración propia.

Otro de los aspectos a considerar fue la estimación y proyección de la tasa de costo de capital doméstica ajustada al riesgo sistémico del proyecto. Para estimar la tasa del costo del capital en dólares, se utilizó el clásico modelo CAPM (capital asset pricing model) de manera aditiva, es decir, apilando primas por riesgo país, y estimando el coeficiente beta por comparables (Copeland, Koller \& Murrin, 2000; Damodaran, 2006; Pratt \& Grabowski, 2008; Fernández, 2014; Damodaran, 2015; Milanesi, 2017). La elección de este modelo obedece al hecho de que los riesgos de mercado del proyecto, expresado por la volatilidad

12 Suponiendo que los riesgos políticos y de sistemas se mantienen en los niveles analizados.

13 Fuente: https://es.investing.com/commodities/brent-oil-historical-data 
Valuación de un contrato de concesión petrolera: análisis del riesgo, simulación... https://doi.org/10.52292/j.eca.2021.2017

precio del Brent, se encuentran altamente integrados y correlacionados con los mercados internacionales. Adicionalmente, las magnitudes financieras del proyecto son expresadas en dólares estadounidenses y esto hace que la tasa de actualización seleccionada se exprese en la misma moneda. La ecuación correspondiente al costo del capital es la siguiente:

$$
k u(x)_{n, t}=r_{f}+\left(M R P \times \beta_{u}\right)+C R P
$$

Tabla 5. Tasa de costo de capital en dólares estimada por año

\begin{tabular}{|c|c|c|c|c|c|}
\hline Periodo & $\begin{array}{l}\text { Tasa libre de } \\
\text { riesgo } \\
\text { EE. UU. (rf) }\end{array}$ & $\begin{array}{l}\text { Country Risk } \\
\text { Premium } \\
(2 / 2020)(\mathrm{CRP})\end{array}$ & $\begin{array}{c}\text { MRP } \\
\text { USD } \\
\text { Argentina }\end{array}$ & $\begin{array}{c}\mathrm{B}(\mathrm{u}) \\
\text { Comparable }\end{array}$ & $\begin{array}{c}\text { Costo Capital } \\
\text { en USD }\end{array}$ \\
\hline 1 & $2,73 \%$ & $8,88 \%$ & $14,08 \%$ & 1,08 & $26,82 \%$ \\
\hline 2 & $2,27 \%$ & $8,88 \%$ & $14,08 \%$ & 1,08 & $26,36 \%$ \\
\hline 3 & $2,23 \%$ & $8,88 \%$ & $14,08 \%$ & 1,08 & $26,32 \%$ \\
\hline 4 & $2,24 \%$ & $8,88 \%$ & $14,08 \%$ & 1,08 & $26,33 \%$ \\
\hline 5 & $2,23 \%$ & $8,88 \%$ & $14,08 \%$ & 1,08 & $26,32 \%$ \\
\hline 6 & $2,20 \%$ & $8,88 \%$ & $14,08 \%$ & 1,08 & $26,29 \%$ \\
\hline 7 & $2,00 \%$ & $8,88 \%$ & $14,08 \%$ & 1,08 & $26,09 \%$ \\
\hline 8 & $2,00 \%$ & $8,88 \%$ & $14,08 \%$ & 1,08 & $26,09 \%$ \\
\hline 9 & $2,00 \%$ & $8,88 \%$ & $14,08 \%$ & 1,08 & $26,09 \%$ \\
\hline 10 & $2,00 \%$ & $8,88 \%$ & $14,08 \%$ & 1,08 & $26,09 \%$ \\
\hline
\end{tabular}

Fuente: elaboración propia.

La tabla 5 proyecta la tasa para los 10 próximos períodos. Respecto del coeficiente beta, se supone que el proyecto se financia íntegramente con capital propio, por lo tanto es utilizado el beta desapalancado del sector (), para el sector Oil/Gas exploración y producción del sitio http:/ / pages.stern. nyu.edu/ adamodar/New_Home_Page/datafile/Betas.html. El adicional 
por riesgo de mercado y el riesgo país es calculado a través del modelo de Damodaran (2015), obtenido del sitio http:/ / pages.stern.nyu.edu/ adamodar/New_Home_Page/datafile/ctryprem.html ${ }^{14}$. El tipo sin riesgo se supone que sigue la curva de inflación proyectada para Estados Unidos, obtenida de https:/ / es.statista.com/estadisticas/598528/ proyeccion-inflacion-en-ee-uu. html. El modelo supone que los niveles de riesgo país, adicional por riesgo de mercado, ambos expresados en dólares, como el coeficiente beta sectorial se mantendrán constante en el tiempo ${ }^{15}$.

\section{Resultados. Diferentes métodos de valuación y análisis del riesgo}

La presente sección expone los resultados obtenidos aplicando diferentes modelos de valuación. Primero es utilizada la técnica de múltiplos y comparables. Seguidamente, se proyectan sobre base determinísticas, los flujos de fondos libres, estimando el valor descontado y tasas de rendimientos. Los resultados de esta sección son tomados como base para el estudio del riesgo del proyecto: equilibrio financiero, sensibilidad, escenarios y simulación empleando las herramientas de la planilla de cálculo. Finalmente, se procede a la valuación de la flexibilidad estratégica contenida en el contrato mediante el enfoque de opciones reales con volatilidad cambiante.

\subsection{Valuación mediante múltiplos y comparables}

El múltiplo utilizado es la relación valor de la empresa, conocida como enterprise value (EV) y EBITDA (ganancias antes de intereses, impuestos, depreciaciones y amortizaciones). El múltiplo es igual a:

14 El modelo empleado construye la tasa utilizando la metodología propuesta por Damodaran (2020), en este caso el riesgo país surge de la calificación dada por Moody, y el adicional por riesgo de mercado doméstico, surge de ajustar el adicional para EE. UU. por el cociente de volatilidad relativa de rendimientos en dólares renta variable argentina sobre renta variable EE. UU., a saber: MRPArg=MRPEE.UUEEUUEEUU.

15 Una de las tantas críticas al modelo CAPM en todas sus manifestaciones reside en su visión de único período, como en la consideración de riesgo sistemático, valuando para inversores eficiente diversificados, abandonando la visión gerencial de la varianza y por ende riesgo del proyecto (Copeland, Weston \& Shastri, 2004; Dempsey, 2013). 
Valuación de un contrato de concesión petrolera: análisis del riesgo, simulación... https://doi.org/10.52292/j.eca.2021.2017

$$
m=E V / E B I T D A
$$

La medida de EBITDA se obtuvo a partir de la producción promedio anual por pozo estimada para los primeros cinco años.

Tabla 6. EBITDA promedio anual

\begin{tabular}{|c|c|}
\hline \multicolumn{2}{|c|}{ Producción total estimada por pozo (máximos) } \\
\hline Plazo inicial concesión & 5 \\
\hline Producción estimada por pozo Bbl acumulada 5 años & 981.240 \\
\hline Producción estimada por pozo Bbl promedio anual & 196.248 \\
\hline Total producción estimada Bbl 4 pozos acumulada 5 años & 3.924 .960 \\
\hline Total producción estimada promedio anual Bbl 4 pozos & 784.992 \\
\hline Ingresos y costos por $\mathrm{Bbl}$ & Dólares EE. UU. \\
\hline Precio barril Brent 5 años promedio & $\$ 58,42$ \\
\hline Costo de desarrollo 3-2020 & $\$ 10,50$ \\
\hline Costo extracción (lifting cost) 3-2020 & $\$ 12,51$ \\
\hline Regalías e impuestos 3-2020 & $\$ 10,00$ \\
\hline Break even & $\$ 33,01$ \\
\hline EBITDA por Bbl al 3-2020 & $\$ 25,41$ \\
\hline EBITDA promedio producción total 4 pozos Bbl. al 2020 & $\$ 19.948 .448,34$ \\
\hline
\end{tabular}

Fuente: elaboración propia.

El múltiplo surge del EBITDA correspondiente al sector Oil/Gas exploración y producción. 
Tabla 7. Múltiplos EBITDA

\begin{tabular}{c|c|c|c|c}
\hline \multirow{2}{*}{ Múltiplos } & \multirow{2}{*}{$\begin{array}{c}\text { Number of } \\
\text { firms }\end{array}$} & \multicolumn{3}{|c}{ Only positive EBITDA firms } \\
\cline { 3 - 5 } Sector & & $\begin{array}{c}\text { EV/ } \\
\text { EBITDAR\&D }\end{array}$ & $\begin{array}{c}\text { EV/ } \\
\text { EBITDA }\end{array}$ & $\begin{array}{c}\text { EV/ } \\
\text { EBIT }\end{array}$ \\
\hline $\begin{array}{c}\text { Oil/Gas (Production and } \\
\text { Exploration) }\end{array}$ & 269 & 4,89 & 4,89 & 13,29 \\
\hline
\end{tabular}

Fuente: http://pages.stern.nyu.edu/ adamodar/New_Home_Page/datafile/vebitda.html.

A partir del múltiplo EV/EBITDA, el valor de la firma asciende a 97.640.694 USD (4,89 x 19.948.448 USD). Este método ofrece un conjunto de desventajas en relación, ya que supone perfecta comparabilidad e integración entre firmas del sector y sistemas económicos, relaciona cifras contables con precios de mercado, que poco tienen que ver con la estimación de valor, entendido este como un proceso subjetivo de proyección y cuantificación de variables estocásticas. En tal sentido, supone comportamientos lineales y determinísticos, en este caso, la firma vale 4,89 veces su EBITDA. Quizá a su favor se puede argumentar la simplicidad de cálculo y, por ello, su empleo difundido, como medida primigenia en la aproximación de valor.

\subsection{Proyección de los flujos de fondos, cálculo del valor actual y tasa de rendimiento}

Se exponen las proyecciones relativas a los flujos de fondos determinísticos del proyecto. La producción es la variable proyectada suponiendo que el precio por barril se mantiene en los promedios de los últimos cinco y diez años, respectivamente. La tabla correspondiente a la proyección para el horizonte explícito (10 años) y su valor de continuidad se presenta en la tabla A.1 del apéndice. A continuación, se presentan los valores actuales y tasas de rendimiento para el horizonte explícito de 10 años. 
Valuación de un contrato de concesión petrolera: análisis del riesgo, simulación... https://doi.org/10.52292/j.eca.2021.2017

Tabla 8. Valores actuales y tasas de rendimiento

\begin{tabular}{lc}
\hline \multicolumn{2}{c}{ Sin valor de continuidad } \\
\hline Valor Actual FFL Brent promedio 5 & $\$ 36.260 .341,57$ \\
\hline Valor Actual FFL Brent promedio 10 & $\$ 66.390 .093,60$ \\
\hline Valor Actual Neto FFL Brent promedio 5 & $\$-25.411 .749,95$ \\
\hline Valor Actual Neto FFL Brent promedio 10 & $\$ 4.718 .002,09$ \\
\hline Inversión + Valor Actual FFL Brent promedio 5 & $\$ 97.932 .433,08$ \\
\hline Inversión + Valor Actual FFL Brent promedio 10 & $\$ 128.062 .185,12$ \\
\hline TIR FFL Brent promedio 5 & $4,36 \%$ \\
\hline TIR FFL Brent promedio 10 & $30,65 \%$ \\
\hline
\end{tabular}

Fuente: elaboración propia.

La vida útil fue estimada en 20 años, los 10 años siguientes al horizonte explícito de proyección representan el valor de continuidad, conocido como steady state, escenario de estabilidad en el comportamiento de las variables. La producción estimada para el décimo período se mantendrá constante durante la vida asignada al proyecto, y como consecuencia de ello es utilizado el último flujo de fondos. Adicionalmente, se supone que la tasa de actualización en dólares ascenderá al $8 \%$ anual, esta surge de la suma entre el tipo libre de riesgo para Estados Unidos y el promedio de los niveles riesgo país para argentina $\left(r f\left(_{E E . U U)} 2 \%+600\right.\right.$ puntos básicos). La anualidad equivalente para $\mathrm{t}=10 \mathrm{es}$ de 6,71008 ${ }^{16}$, arrojando un valor de continuidad de 19 millones de dólares estadounidenses (Brent 5 años promedio) y 36 millones de dólares estadounidenses (Brent 10 años promedio).

$\overline{16} \quad \mathrm{~A}=(1+\mathrm{k}) \mathrm{t}-1(1+\mathrm{k}) \mathrm{tk}$. 
Tabla 9. Valores actuales y valor de continuidad

\begin{tabular}{l|r}
\hline \multicolumn{2}{c}{ Valor de continuidad } \\
\hline Valor Continuidad FFL Brent promedio 5 en $\mathrm{t}=10$ & $\$ 19.820 .941,99$ \\
\hline Valor Continuidad FFL Brent promedio 10 en $\mathrm{t}=10$ & $\$ 36.585 .654,02$ \\
\hline Valor Continuidad FFL Brent promedio 5 en $\mathrm{t}=0$ & $\$ 1.490 .086,62$ \\
\hline Valor Continuidad FFL Brent promedio 10 en $\mathrm{t}=0$ & $\$ 2.750 .413,87$ \\
\hline Valor Actual + Valor Continuidad FFL Brent promedio 5 & $\$ 37.750 .428,19$ \\
\hline Valor Actual + Valor Continuidad FFL Brent promedio 10 & $\$ 69.140 .507,47$ \\
\hline Valor Actual Neto FFL Brent promedio 5 & $\$-23.921 .663,32$ \\
\hline Valor Actual Neto FFL Brent promedio 10 & $\$ 7.468 .415,95$ \\
\hline
\end{tabular}

Fuente: elaboración propia.

A diferencia de una empresa en marcha, el valor de continuidad no representa un valor significativo del total correspondiente al intrínseco de los flujos, debido a su horizonte limitado (Copeland, Weston \& Shastri, 2004). En este caso, asciende al $4 \%$ del total.

De los resultados obtenidos se puede observar el impacto que el precio del crudo tiene sobre los valores proyectados, al comparar resultados tomando como precio el barril Brent, tanto el promedio 5 y 10 años. No obstante, el flujo de fondos determinístico no permite capturar e incorporar en el valor intrínseco la flexibilidad estratégica contenida en el pliego del contrato, ejercible en el quinto y décimo año.

\subsection{Análisis de riesgo}

El análisis del riesgo se efectúo tomando como base los flujos de fondos libres proyectados sobre la base del precio promedio del barril Brent en los últimos cinco años. A continuación, los diferentes métodos. 


\subsubsection{Punto de equilibrio financiero}

Esta herramienta permite realizar un análisis de sensibilidad de única variable, con el fin de determinar las cantidades de equilibrio (barriles) a producir para que el valor actual neto sea igual a cero. Para ello es necesario que se cumpla la siguiente condición:

$$
I=F F L(E) \times \frac{\left(1+k_{u}\right)^{t}-1}{\left(1+k_{u}\right)^{t} \times k_{u}}
$$

Donde representa la inversión inicial compuesta por el activo fijo más el capital de trabajo, la tasa de costo de capital desapalancado, $t$ el horizonte de proyección y la corriente de flujos de fondos libres equivalentes que iguala a la inversión, logrando la condición de valor actual neto igual a cero. Obtenido el flujo de fondos libres equivalente, se procede a dividir por la contribución marginal promedio, arrojando las cantidades de equilibrio.

Tabla 10. Punto de equilibrio financiero

\begin{tabular}{l|r}
\hline \multicolumn{2}{|c}{ Punto de equilibrio financiero } \\
\hline Inversión inicial & $\$ 61.361 .398,22$ \\
\hline Tasa promedio en dólares & $26,5 \%$ \\
\hline Anualidad $(1+\mathrm{k}) \mathrm{n}-1 /(1+\mathrm{k}) \mathrm{n}{ }^{*} \mathrm{k}$ & $\$ 3,41$ \\
\hline Flujos de Fondos Libres Equivalente FFL(E) & $\$ 17.971 .236,97$ \\
\hline Contribución marginal & $\$ 25,41$ \\
\hline PE: Barriles por año promedio & 707.187 \\
\hline
\end{tabular}

Fuente: elaboración propia.

En este caso se tomó una tasa promedio en dólares (26,5\%) y el equilibrio se da con 707.187 barriles producidos, cifra de producción obtenida entre el primer y tercer año, ya que a partir del cuarto la cantidad de barriles se reduce sensiblemente. 


\subsubsection{Análisis de sensibilidad}

El primer análisis de sensibilidad realizado es de única variable, manteniendo el resto de las variables constantes, se procedió a sensibilizar de forma individual precio barril Brent, y costo del capital, fijando como restricción un valor actual neto igual a cero. El proceso se realizó utilizando la herramienta del programa MS Excel ${ }^{\circledR}$ datos, análisis de hipótesis, buscar objetivo ${ }^{17}$.

Tabla 11. Sensibilidad única variable precio barril, costo capital

Sensibilidad única variable

Precio Brent (variable iterada)

Costo Capital promedio (variable iterada)

$4,08 \%$

Fuente: elaboración propia.

Suponiendo el resto constante, el precio del crudo debería subir a 71 USD por barril, o la tasa de costo de capital disminuir al 4,08\% (equivalente a la tasa interna de retorno expuesta en la tabla 8). Seguidamente, se expone el análisis de sensibilidad de dos variables con la herramienta del programa MS Excel $®$ datos, análisis de hipótesis, tabla de datos ${ }^{18}$.

Las 78 observaciones generadas por la tabla son resumidas en estadísticos descriptivos complementados con las siguientes medidas:

$$
V E=\sum_{i=1}^{n} \quad V_{i} \times p_{i}
$$

La ecuación presenta el valor esperado como la suma ponderada entre el valor obtenido en cada iteración y su probabilidad de ocurrencia, en este caso $1 / \mathrm{n}$ por resultado.

17 @Buscar objetivo, definir celda objetivo (VAN), con valor (restricción), iterando (variable seleccionada) (Benninga, 2008; Machain, 2011).

$18 @$ Tabla de datos, contenido en el menú Datos, Análisis de hipótesis. Se debe ubicar en el extremo el resultado objetivo a sensibilizar (VAN), e indicar la celda desde donde se tomará el valor de filas (precio Brent) y las columnas (tasa costo capital), sombreando el área seleccionada (Benninga, 2008; Machain, 2011). 
Valuación de un contrato de concesión petrolera: análisis del riesgo, simulación... https://doi.org/10.52292/j.eca.2021.2017

\begin{tabular}{|c|c|c|c|c|c|c|}
\hline $\begin{array}{l}8 \\
0 \\
010 \\
0\end{array}$ & 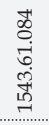 & 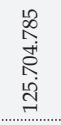 & 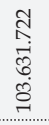 & 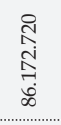 & 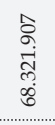 & $\begin{array}{l}\text { हे } \\
\dot{\delta} \\
\tilde{\sigma} \\
\text { ठे }\end{array}$ \\
\hline $\begin{array}{l}\stackrel{8}{0} \\
\text { o्? }\end{array}$ & $\begin{array}{l}\infty \\
\stackrel{0}{0} \\
\sigma \\
0 \\
0 \\
\stackrel{2}{2}\end{array}$ & 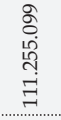 & 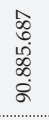 & 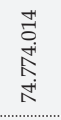 & 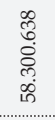 & 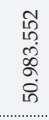 \\
\hline $\begin{array}{l}8 \\
\text { ถু }\end{array}$ & 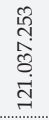 & $\begin{array}{l}m \\
\overrightarrow{7} \\
\ddot{8} \\
0 \\
0 \\
0\end{array}$ & $\begin{array}{l}\overrightarrow{0} \\
0 \\
0 \\
0 \\
\infty \\
\infty\end{array}$ & 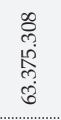 & 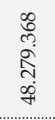 & 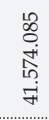 \\
\hline $\begin{array}{l}8 \\
\stackrel{\circ}{\circ}\end{array}$ & 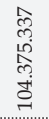 & 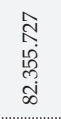 & $\begin{array}{l}n \\
\overrightarrow{0} \\
\infty \\
0 \\
03 \\
030\end{array}$ & 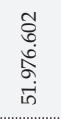 & $\begin{array}{l}\text { oे } \\
\text { oे } \\
\infty \\
0 \\
0 \\
\infty \\
\text { co }\end{array}$ & 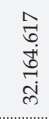 \\
\hline $\begin{array}{l}8 \\
10 \\
\infty 1\end{array}$ & 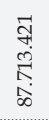 & 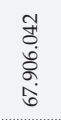 & 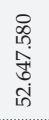 & 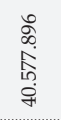 & 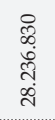 & 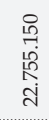 \\
\hline $\begin{array}{l}8 \\
\stackrel{1}{\infty} \\
\infty\end{array}$ & 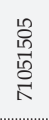 & 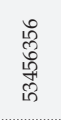 & $\begin{array}{l}\text { 苦 } \\
\text { 离 } \\
\text { ले }\end{array}$ & 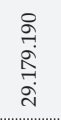 & 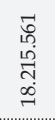 & 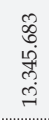 \\
\hline $\begin{array}{l}8 \\
1 \\
k\end{array}$ & 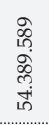 & 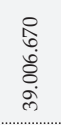 & 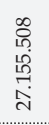 & 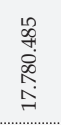 & $\begin{array}{l}\text { నิ } \\
\text { ఫे } \\
\stackrel{\infty}{\infty}\end{array}$ & $\begin{array}{l}\text { ते } \\
\text { ळूँ } \\
\text { ले }\end{array}$ \\
\hline $\begin{array}{l}\stackrel{8}{\circ} \\
\stackrel{2}{2}\end{array}$ & 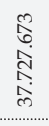 & 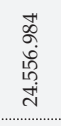 & 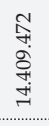 & 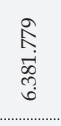 & 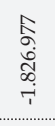 & 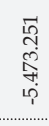 \\
\hline $\begin{array}{l}8 \\
16 \\
16\end{array}$ & 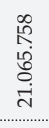 & $\begin{array}{l}\infty \\
\stackrel{1}{ } \\
0 \\
0 \\
0 \\
0\end{array}$ & 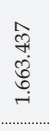 & 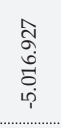 & 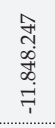 & $\begin{array}{l}\text { ते } \\
\text { ते } \\
0 \\
\infty \\
+ \\
+\end{array}$ \\
\hline $\begin{array}{l}8 \\
8 \\
8\end{array}$ & 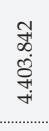 & 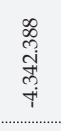 & $\begin{array}{l}\text { oे } \\
\text { त. } \\
0 \\
0 \\
\overrightarrow{7} \\
\overrightarrow{7}\end{array}$ & 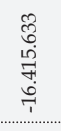 & 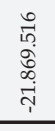 & 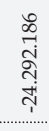 \\
\hline $\begin{array}{l}\text { fี } \\
\text { ơ } \\
\text { in }\end{array}$ & $\begin{array}{l}\text { त } \\
\text { ते } \\
\overrightarrow{0} \\
\infty\end{array}$ & 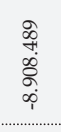 & 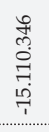 & 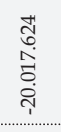 & 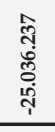 & 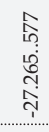 \\
\hline $\begin{array}{l}8 \\
\text { in } \\
\text { in }\end{array}$ & 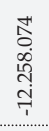 & 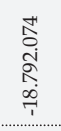 & 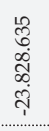 & 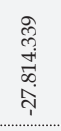 & 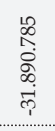 & 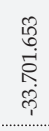 \\
\hline $\begin{array}{l}8 \\
\text { î̀ }\end{array}$ & 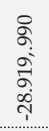 & 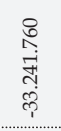 & 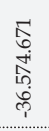 & 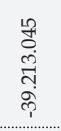 & $\begin{array}{l}\text { 艹艹 } \\
\text { जे } \\
\vec{J} \\
\vec{F}\end{array}$ & 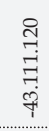 \\
\hline 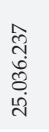 & $\begin{array}{l}\stackrel{0}{0} \\
8 \\
\text { in }\end{array}$ & $\begin{array}{l}\stackrel{2}{0} \\
\stackrel{8}{0} \\
\stackrel{0}{0}\end{array}$ & $\begin{array}{l}\circ \\
\stackrel{8}{0} \\
\stackrel{2}{0}\end{array}$ & $\begin{array}{l}\stackrel{0}{\circ} \\
\stackrel{\leftrightarrow}{0}\end{array}$ & $\begin{array}{l}\text { ஃ̊ } \\
\text { గn్ } \\
\text { ڤ్ర }\end{array}$ & $\begin{array}{l}\stackrel{0}{0} \\
\stackrel{0}{0} \\
\stackrel{0}{0}\end{array}$ \\
\hline
\end{tabular}




$$
V E G P=\sum_{i=1}^{n} \quad V_{i}^{+} \times p_{i}
$$

La ecuación presenta el valor esperado de las ganancias perdidas, es decir el costo de rechazar el proyecto. Surge de la suma ponderada entre el valor obtenido positivo en cada iteración y su probabilidad de ocurrencia, en este caso $1 / \mathrm{n}$ por resultado

$$
V E P E=\sum_{i=1}^{n} \quad V_{i}^{-} \times p_{i}
$$

El valor esperado de las pérdidas evitadas representa el beneficio de rechazar el proyecto. Surge de la suma ponderada entre el valor obtenido negativo en cada iteración y su probabilidad de ocurrencia, en este caso $1 / \mathrm{n}$ por resultado

$$
R P E=\frac{|V E P E|}{V E P E+|V E P E|}
$$

Ratio pérdida esperada indica sobre el valor total de posibles resultados el beneficio de rechazar el proyecto.

Tabla 13. Estadísticos y resumen de resultados

\begin{tabular}{l|r}
\hline Media & $\$ 27.597 .336,71$ \\
\hline Desvío & $\$ 47.961 .990,60$ \\
\hline CV & 0,575400153 \\
\hline Valor esperado (VE) & $\$ 27.597 .336,71$ \\
\hline VE ganancias perdidas (VEGP) & $\$ 35.153 .643,98$ \\
\hline VE pérdidas evitadas (VEPE) & $\$-7.556 .307,27$ \\
\hline Ratio pérdida esperada (RPE) & $17,69 \%$ \\
\hline Decisión & Acepta \\
\hline
\end{tabular}

Fuente: elaboración propia. 
Valuación de un contrato de concesión petrolera: análisis del riesgo, simulación... https://doi.org/10.52292/j.eca.2021.2017

Para el caso bajo estudio, la ganancia obtenida por rechazar el proyecto representa un 17,69 \% de los beneficios totales. El complemento implica asumir el costo de oportunidad de rechazar un proyecto con valor positivo, conduciendo a ser aceptado.

\subsubsection{Análisis de escenarios}

Los escenarios fueron planteados suponiendo comportamientos para las variables precio del crudo, estructuras de costos y tasa de costo de capital (Miller \& Waller, 2003; Esandi, Milanesi \& Pesce, 2012). Para ello se utilizó el administrador de escenario contenido en el programa MS Excel ${ }^{19}$.

Seguidamente, fueron asignadas probabilidades subjetivas a cada uno de los escenarios generados, obteniéndose la siguiente tabla de resultados (tabla 15).

A diferencia de la tabla, en este caso el juicio del experto a partir del estudio del contexto, la proyección de las variables y la asignación de probabilidades subjetivas ${ }^{20}$, hace que el proyecto sea rechazado y deban extremarse las técnicas de estudio. El ratio de pérdida esperada asciende a un 53,5\%, un resultado ambiguo y no concluyente sobre la viabilidad del proyecto.

\subsubsection{Simulación}

La simulación Montecarlo se desarrolló empleando el programa MS Excel ${ }^{\circledR}$, básicamente trabajando con el comando de generación de números aleatorios (Benninga, 2008; Machain, 2011). Las variables de entrada seleccionadas fueron:

a. Precio del crudo, suponiendo que sigue un proceso estocástico con distribución normal ${ }^{21}$, cuyos parámetros son media: 58, 42 USD, desvío: 27,9 USD en función a la serie histórica correspondientes a los últimos cinco años.

19 @Administrador de escenario, contenido en el menú Datos, Análisis de hipótesis. Por escenario se deben cargar las variables y asignar los respectivos valores, para el caso estudiado fue: precio Brent, costos de desarrollo, costos de explotación, tasa de descuento. Seguidamente, se debe fijar la celda de resultado, en este caso el valor actual neto. La salida es un tabla resumen con las celdas de cada escenario y el resultado obtenido (Benninga, 2008; Machain, 2011).

20 Sin perjuicio de los sesgos y heurísticas que puedan atravesar su juicio en la toma de decisiones en condiciones de incertidumbre (El Alabi \& Milanesi, 2016).

21 La función es @inv.norm(aleatorio; media; desvío). 
Gastón Silverio Milanesi

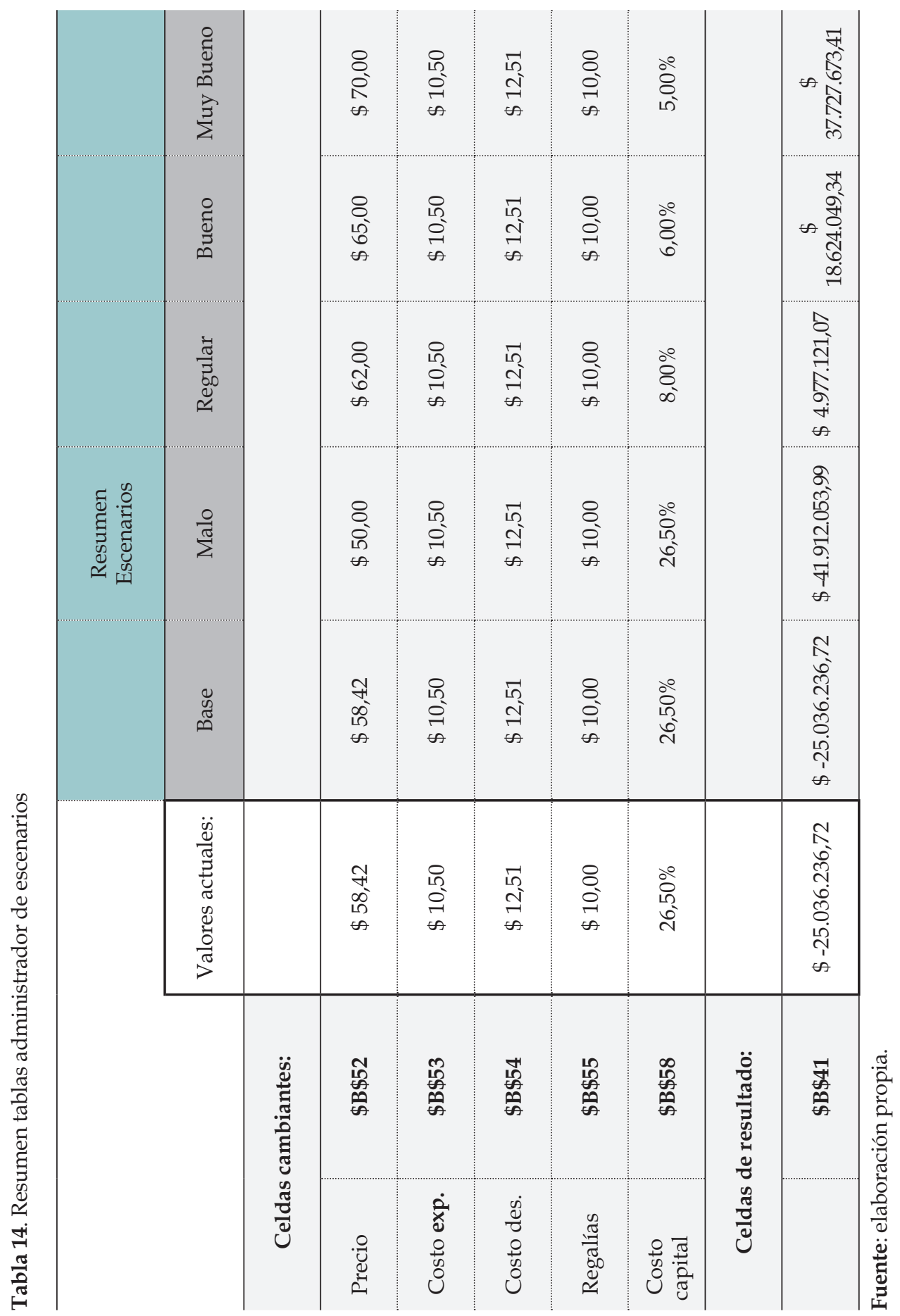


Valuación de un contrato de concesión petrolera: análisis del riesgo, simulación... https://doi.org/10.52292/j.eca.2021.2017

Tabla 15. Valor esperado escenarios y criterios de elección

\begin{tabular}{|c|c|c|c|c|c|}
\hline \multirow{2}{*}{ Escenario } & \multirow{2}{*}{ Probabilidad } & \multirow{2}{*}{ Valor } & \multirow{2}{*}{ Valor probable } & \multicolumn{2}{|c|}{ Criterios } \\
\hline & & & & VE & \$-1.624.867,97 \\
\hline $\begin{array}{c}\text { Muy } \\
\text { Bueno }\end{array}$ & $16,50 \%$ & $\$ 37.727 .673,41$ & $\$ 6.225 .066,11$ & Desvío & $\$ 27.429 .807,86$ \\
\hline Bueno & $21,50 \%$ & \$ 18.624.049,34 & $\$ 4.004 .170,61$ & $\mathrm{CV}$ & $\$ 16,88$ \\
\hline Regular & $21,50 \%$ & $\$ 4.977 .121,07$ & $\$ 1.070 .081,03$ & VEGP & \$ 11.299.317,75 \\
\hline Base & $24,00 \%$ & $\$-25.036 .236,72$ & \$-6.008.696,81 & VEPE & \$-12.924.185,72 \\
\hline Malo & $16,50 \%$ & $\$-41.912 .053,99$ & \$ -6.915.488,91 & RPE & $53,35 \%$ \\
\hline Total & $100,00 \%$ & Valor esperado & $\$-1.624 .867,97$ & Decisión & Rechaza \\
\hline
\end{tabular}

Fuente: elaboración propia.

b. Tasa de actualización, suponiendo que sigue un proceso estocástico con distribución normal ${ }^{22}$, cuyos parámetros son media: $10 \%$, desvío: $5 \%$, en función a parámetros estables de tasa en dólares para Argentina.

c. Cantidad de barriles, se supone una distribución uniforme con producción variable por año ${ }^{23}$.

Las variables de salida son:

a. Valor actual neto de los flujos de fondos:

b. Tasa interna de retorno modificada, suponiendo la tasa del costo del capital como factor de reinversión y financiamiento ${ }^{24}$.

c. Rendimiento logarítmico del proyecto en el primer año, , siendo el valor actual de los flujos de fondos. En el caso del valor actual de los flujos de fondos en el momento $t=0$, se descuenta la totalidad de la corriente de flujos proyectados. Los estadísticos descriptivos correspondiente a

22 La función es @inv.norm(aleatorio; media; desvío).

23 La función es @aleatorio.entre $(. . . ; \ldots . .$.$) .$

24 La función es @TIRM(tasa de financiamiento; tasa de reinversión, serie de datos) 
la simulación de esta variable, en particular el desvío estándar, sirve para calcular la volatilidad del subyacente en la valoración de opciones mediante el enfoque MAD (marketed asset disclaimer) (Copeland \& Antikarov, 2003; Smith, 2005; Brandao, Dyer \& Hahnn, 2012; Pareja Vasseur, Prada Sánchez \& Moreno Escobar, 2019).

El número de iteraciones para cada variable de salida fue planteado en $2000^{25}$. A título ilustrativo se presenta la pantalla de la planilla de cálculo con las iteraciones.

Figura 1. Salidas e iteraciones simulación MS Excel ${ }^{\circledR}$

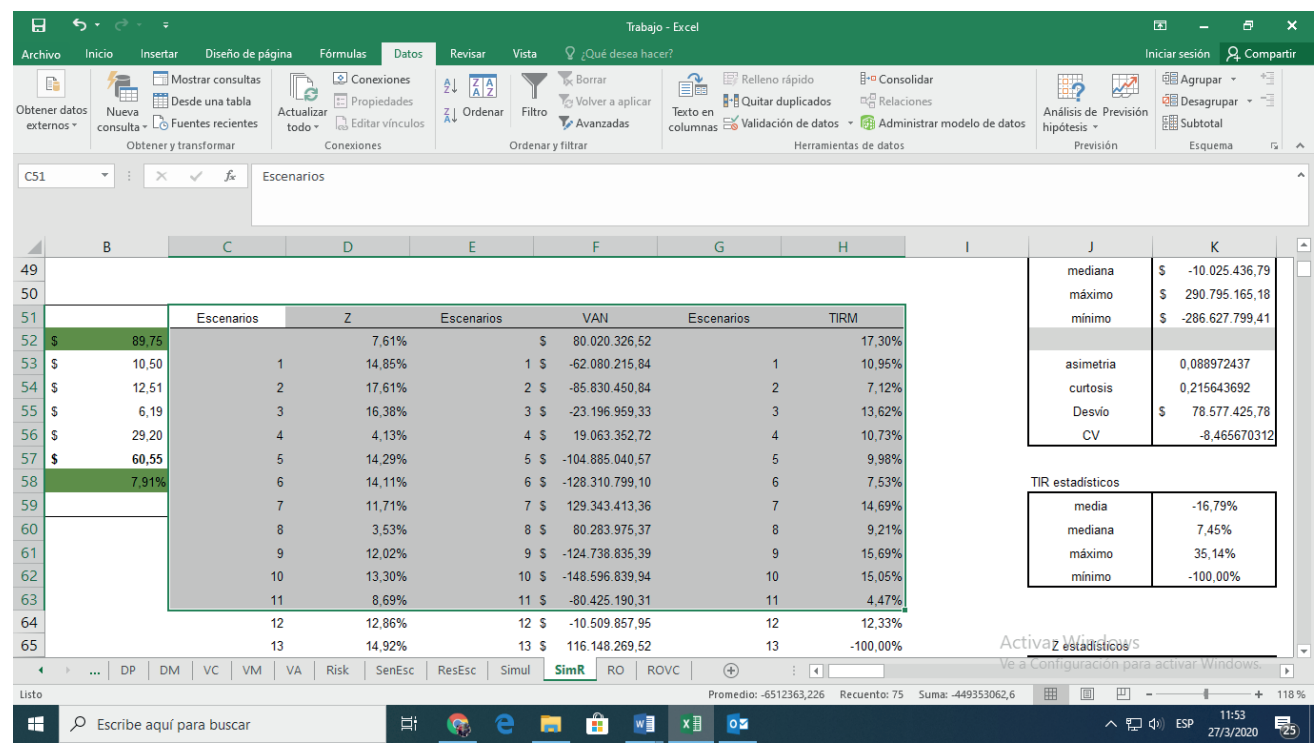

Fuente: elaboración propia.

25 Plantados el número de iteraciones en una columna de planilla de cálculo, la otra columna debe reservarse para copiar la referencia a la celda de salida. Seguidamente se sombre las columnas (número de observaciones, y la columna en blanco con la primera celda, que toma el valor de la variable de salida), en el menú Datos, Análisis de hipótesis, Tabla de datos, se deja la celda fila en blanco, la columna se referencia a la celda en blanco, inmediata superior, a la celda de valor de salida. El programa copia y pega ese valor en cada celda que representa un escenario (de 1 a n), a medida que realiza esta acción la función @aleatorio () genera valores aleatorios para las variables de entrada, de acuerdo al proceso estocástico especificado. En el ejemplo el proceso se repitió 2000 veces (Benninga, 2008); (Machain, 2011). 
Valuación de un contrato de concesión petrolera: análisis del riesgo, simulación... https://doi.org/10.52292/j.eca.2021.2017

\section{Seguidamente, se exponen la salida de los principales estadísticos para el Valor Actual Neto.}

Tabla 16. Estadísticos Valor Actual Neto. Simulación

\begin{tabular}{cc}
\hline Media & $\$-9.281 .890,61$ \\
\hline Mediana & $\$-10.025 .436,79$ \\
Máximo & $\$ 290.795 .165,18$ \\
Mínimo & $\$-286.627 .799,41$ \\
Asimetria & 0,088972437 \\
Curtosis & 0,215643692 \\
Desvío & $\$ 78.577 .425,78$ \\
CV & $-8,465670312$ \\
\hline
\end{tabular}

Fuente: elaboración propia.

Con los datos de la media y el desvío se estandariza $(z)$ con el fin de obtener la probabilidad de un VAN mayor a cero y mayor a 10.0000.000 USD.

Tabla 17. Distribución normal y probabilidades de VAN $>=0, \mathrm{VAN}>=10.000 .000$

\begin{tabular}{|c|c|c|c|c|c|c|}
\hline \multirow{2}{*}{$z=(x-\mu) / \sigma$} & \multirow{2}{*}{$\begin{array}{c}\text { Variable } \\
x\end{array}$} & \multirow{2}{*}{$\begin{array}{c}\begin{array}{c}\text { Estandariza- } \\
\text { ción }\end{array} \\
\mathrm{z}\end{array}$} & \multicolumn{2}{|c|}{$\begin{array}{c}\text { 1-Prob }(Z<Z o) \text { a la izquierda de } \\
\text { la curva }\end{array}$} & \multicolumn{2}{|c|}{$\operatorname{Prob}(Z<Z o)$ a la izquierda de la curva } \\
\hline & & & $\begin{array}{l}\text { 1-Distr.Norm. } \\
\text { Estand }(\mathrm{z})^{1}\end{array}$ & $\begin{array}{l}\text { 1-Distr. } \\
\operatorname{Norm}(x, m, d, v-f)^{2}\end{array}$ & $\begin{array}{l}\text { 1-(1-Distr.Norm. } \\
\text { Estand(z)) }\end{array}$ & $\begin{array}{l}\text { 1-(1-Distr. } \\
\operatorname{Norm}(\mathrm{x}, \mathrm{m}, \mathrm{d}, \mathrm{v}-\mathrm{f}))\end{array}$ \\
\hline $\mathrm{P}(\mathrm{x}) \mathrm{VAN} \geq$ & \$- & 0,11 & $45,44 \%$ & $45,44 \%$ & $54,56 \%$ & $54,56 \%$ \\
\hline $\mathrm{P}(\mathrm{x}) \mathrm{VAN} \geq$ & $\$ 10.000 .000$ & 0,2389 & $40,56 \%$ & $40,56 \%$ & $59,44 \%$ & $59,44 \%$ \\
\hline
\end{tabular}

Fuente: elaboración propia.

26 @Distr.norm.estand $(Z)$ devuelve la función de distribución normal estándar acumulativa para la variable $z$, en este caso VAN $(z)>=0$, la función arroja el acumulado hasta $z$, o sea el complemento (54,56\%), 1-@Distr.norm.estand(Z) arroja el valor correspondiente a la condición >=(z).

27 @Distr.Norm (valor variable X, media obtenida, desvío obtenido, Verdadero/Falso) devuelve la función de distribución normal para la media y desvío estándar especificado. Si el argumento media $=0$, desvío=1 y el argumento lógico es Verdadero, devuelve la distr.nom.estand (Z) para la X especificada. En el caso de establecer el argumento verdadero devuelve el área (integral) desde infinito negativo hasta el valor de x (54,56 \%), 1-@Distr.Norm arroja el valor correspondiente a la condición $>=(X)$. 
La tabla de estadísticos para la TIRM son los siguientes.

Tabla 18. Estadísticos TIRM Simulación

\begin{tabular}{c|c}
\hline Media & $-14,72 \%$ \\
\hline Mediana & $8,06 \%$ \\
\hline Máximo & $37,89 \%$ \\
\hline Mínimo & $-100,00 \%$ \\
\hline
\end{tabular}

Fuente: elaboración propia.

La tabla de estadísticos para $\ln (z)$ es:

Tabla 19. Estadísticos $\ln (\mathrm{z})$ Simulación

\begin{tabular}{c|c}
\hline Media & $9,31 \%$ \\
\hline Mediana & $9,41 \%$ \\
\hline Máximo & $23,4 \%$ \\
Mínimo & $-7,23 \%$ \\
\hline Asimetria & $-0,183984688$ \\
\hline Curtosis & 0,007415254 \\
\hline Desvío & $4,55 \%$ \\
\hline CV & 0,488983142 \\
\hline
\end{tabular}

Fuente: elaboración propia.

Los resultados que arroja la simulación obligan a rechazar el proyecto, ya que las probabilidades de VAN superior a cero son inferiores al $50 \%$. Esto tiene su correlato con los resultados obtenidos para la TIRM. Los valores de la variable $\ln (\mathrm{z})$, representa el rendimiento obtenido por cociente entre el valor intrínseco esperado y valor intrínseco actual. Este presenta una media de 9,31 \% y un desvío de 4,55 \%, siendo, esta última, medida de volatilidad en el enfoque MAD tradicional (Copeland \& Antikarov, 2003). 
Valuación de un contrato de concesión petrolera: análisis del riesgo, simulación...

https://doi.org/10.52292/j.eca.2021.2017

\subsection{Opciones reales: el modelo binomial, volatilidad cambiante y MAD para el steady state}

Se procede a valuar el contrato con las opciones incluidas en su respectivo pliego. Para ello se adopta un enfoque binomial de opciones, con volatilidad cambiante y decreciente, estabilizándose a partir del valor de continuidad. El comportamiento estocástico se centra en el precio del barril Brent, suponiéndose que los costos se encuentran correlacionados perfectamente. Para la proyección de la rejilla binomial para el Brent, se proyectaron los siguientes niveles de volatilidad:

Tabla 20. Volatilidad proyectada Brent

\begin{tabular}{c|c|c|c}
\hline $\mathbf{1}$ al 4 & $\mathbf{5}$ al 7 & $\mathbf{8}$ al 10 & $\mathbf{1 0} \rightarrow$ \\
\hline$\sigma 1$ & o2 & $\sigma 3$ & $\sigma 4$ \\
\hline $49,68 \%$ & $43,01 \%$ & $38,68 \%$ & $4,55 \%$ \\
\hline
\end{tabular}

Fuente: elaboración propia.

La tabla 20 supone que los tres niveles de volatilidad replican el comportamiento de la volatilidad histórica expuesta en la tabla 4, estabilizando a partir del año 11 en adelante. En este caso, la volatilidad se supone la obtenida aplicando el enfoque MAD (tabla 19). Con los datos de la tabla 20, y la tasa libre de riesgo expresada en dólares del 2,5 \% anual promedio (tabla 5), se expone la tabla 21 con los movimientos de ascenso y descenso máximos, y los coeficientes equivalentes ciertos para cada estadio de riesgo (ecuaciones 1 a 6).

Tabla 21. Coeficientes y equivalentes ciertos (ecuaciones 1 a 6 )

\begin{tabular}{c|c|c|}
\hline Movimientos & $u \max$ & $d \max$ \\
\hline Coeficientes & 1,740365 & 0,574592 \\
\hline Equiv.Ciertos & $p u$ & $\mathbf{1 - p u}$ \\
\hline 1 al 4 & 0,386360 & 0,613640 \\
\hline 5 al 7 & 0,289566 & 0,710434 \\
\hline 8 al 10 & 0,234193 & 0,765807 \\
\hline 10 en adelante & 0,003292 & 0,996708 \\
\hline
\end{tabular}

Fuente: elaboración propia. 
Seguidamente, se procede a proyectar la rejilla binomial, correspondiente al movimiento estocástico del precio del crudo (ecuación 7),

Tabla 22. Rejilla binomial precio Brent (ecuación 7)

\begin{tabular}{|c|c|c|c|c|c|c|c|c|c|c|}
\hline 0 & 1 & 2 & 3 & 4 & 5 & 6 & 7 & 8 & 9 & 10 \\
\hline \multirow[t]{11}{*}{28,0} & 48,7 & 84,7 & 147,4 & 256,5 & 446,4 & 776,9 & 1352,1 & 2353,2 & 4095,4 & 7127,5 \\
\hline & 16,1 & 28,0 & 48,7 & 84,7 & 147,4 & 256,5 & 446,4 & 776,9 & 1352,1 & 2353,2 \\
\hline & & 9,2 & 16,1 & 28,0 & 48,7 & 84,7 & 147,4 & 256,5 & 446,4 & 776,9 \\
\hline & & & 5,3 & 9,2 & 16,1 & 28,0 & 48,7 & 84,7 & 147,4 & 256,5 \\
\hline & & & & 3,0 & 5,3 & 9,2 & 16,1 & 28,0 & 48,7 & 84,7 \\
\hline & & & & & 1,8 & 3,0 & 5,3 & 9,2 & 16,1 & 28,0 \\
\hline & & & & & & 1,0 & 1,8 & 3,0 & 5,3 & 9,2 \\
\hline & & & & & & & 0,6 & 1,0 & 1,8 & 3,0 \\
\hline & & & & & & & & 0,3 & 0,6 & 1,0 \\
\hline & & & & & & & & & 0,2 & 0,3 \\
\hline & & & & & & & & & & 0,1 \\
\hline
\end{tabular}

Fuente: elaboración propia.

Con el fin de obtener el flujo de fondos libres y el valor del subyacente, son utilizadas las ecuaciones 8 a 11. En primer lugar, el proceso estocástico correspondiente al flujo de fondos operativo después de impuestos se obtiene calculado la proporción del EBIT por barril después de impuestos, sobre cada nodo proyectado para el precio del crudo, . 
Valuación de un contrato de concesión petrolera: análisis del riesgo, simulación... https://doi.org/10.52292/j.eca.2021.2017

Tabla 23. Rejilla binomial Flujo de fondos operativos

\begin{tabular}{|c|c|c|c|c|c|c|c|c|c|c|}
\hline 0 & 1 & 2 & 3 & 4 & 5 & 6 & 7 & 8 & 9 & 10 \\
\hline \multirow[t]{11}{*}{8,5} & 14,8 & 25,8 & 44,9 & 78,1 & 135,9 & 236,6 & 411,7 & 716,5 & 1247,0 & 2170,2 \\
\hline & 4,9 & 8,5 & 14,8 & 25,8 & 44,9 & 78,1 & 135,9 & 236,6 & 411,7 & 716,5 \\
\hline & & 2,8 & 4,9 & 8,5 & 14,8 & 25,8 & 44,9 & 78,1 & 135,9 & 236,6 \\
\hline & & & 1,6 & 2,8 & 4,9 & 8,5 & 14,8 & 25,8 & 44,9 & 78,1 \\
\hline & & & & 0,9 & 1,6 & 2,8 & 4,9 & 8,5 & 14,8 & 25,8 \\
\hline & & & & & 0,5 & 0,9 & 1,6 & 2,8 & 4,9 & 8,5 \\
\hline & & & & & & 0,3 & 0,5 & 0,9 & 1,6 & 2,8 \\
\hline & & & & & & & 0,2 & 0,3 & 0,5 & 0,9 \\
\hline & & & & & & & & 0,1 & 0,2 & 0,3 \\
\hline & & & & & & & & & 0,1 & 0,1 \\
\hline & & & & & & & & & & 0,0 \\
\hline
\end{tabular}

Fuente: elaboración propia.

Los valores obtenidos en la tabla 23 se transforman en totales mediante el producto entre la producción de barriles por año (tabla 1) y el flujo operativo por barril, $F F O(\text { total })_{t}^{i, j}=F F O_{t}^{i, j} \times B b l(t)$. 
Gastón Silverio Milanesi

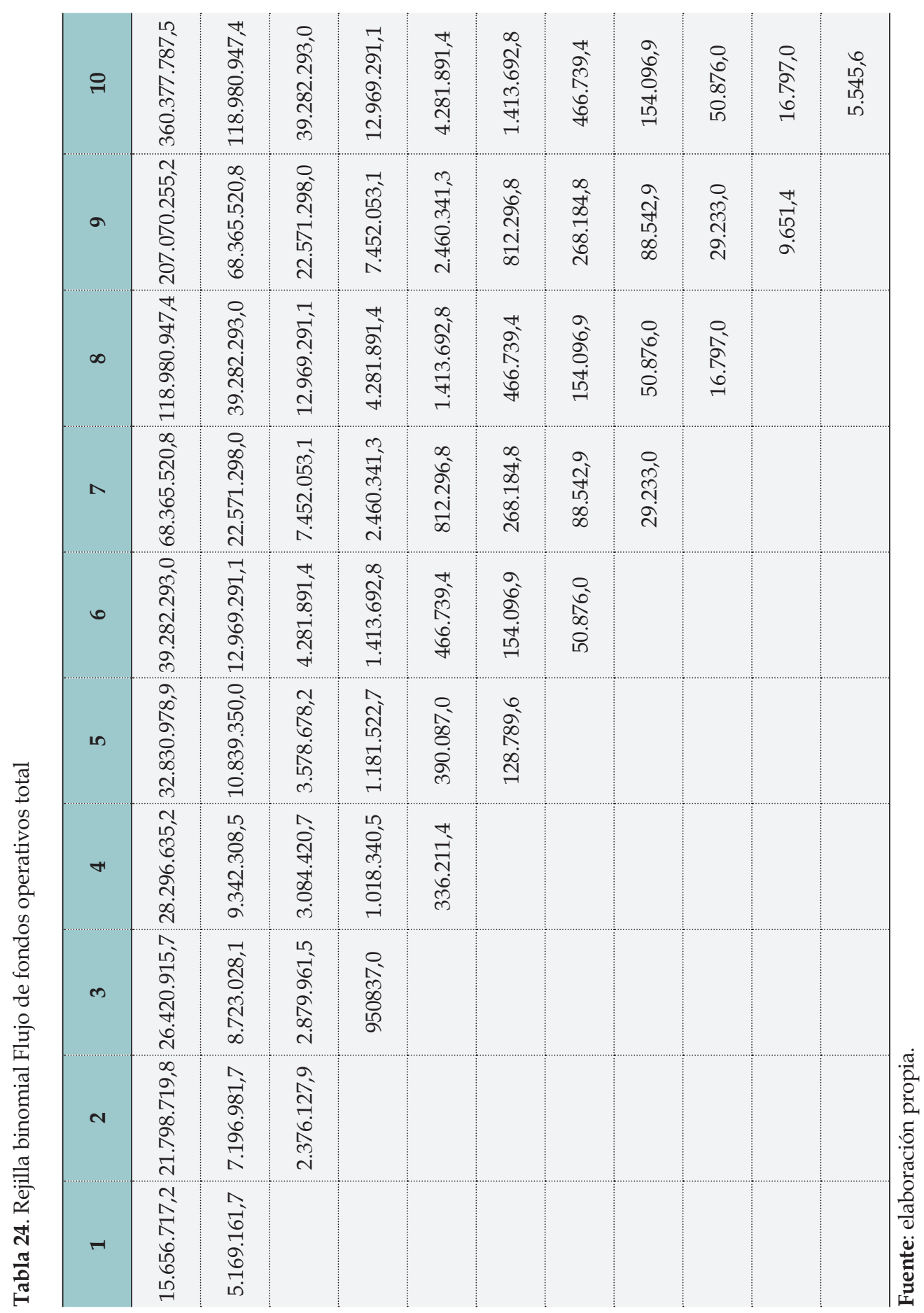


Al flujo de fondos libres se llega descontando del flujo operativo (tabla 24), las inversiones incrementales. Primero, se estima el porcentaje que representa el capital de trabajo incremental sobre el EBIT determinístico, calculado en el anexo 1, tabla 1, para la estimación del valor actual.

Tabla 25: Inversión incremental en capital de trabajo sobre flujo operativo determinístico

\begin{tabular}{c|c|c|c|c|c|c|c|c|c}
\hline 1 & 2 & 3 & 4 & 5 & 6 & 7 & 8 & 9 & 10 \\
\hline $0,000 \%$ & $-2,224 \%$ & $-3,877 \%$ & $-5,560 \%$ & $-4,448 \%$ & $-4,043 \%$ & $0,000 \%$ & $0,000 \%$ & $0,000 \%$ & $0,000 \%$ \\
\hline
\end{tabular}

Fuente: elaboración propia.

Deduciendo el resultado de la inversión incremental en capital de trabajo del flujo operativo se obtiene la rejilla binomial. Para cada nodo .

La rejilla para la estimación del proceso recursivo y valoración de las opciones requiere, en primer lugar, suponer que a partir del año 10 hasta el 20 se estabilizan la volatilidad y el crecimiento. El valor actual de la firma en cada uno de los nodos correspondientes al décimo período se obtiene por el producto entre el flujo de fondos estimado para cada nodo del décimo año y el valor de la anualidad para $t=10$ hasta 2 . En este caso, siendo, según la tabla 27. 
Gastón Silverio Milanesi

\begin{tabular}{|c|c|c|c|c|c|c|c|c|c|c|c|}
\hline 으 & 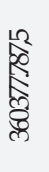 & 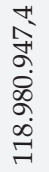 & $\begin{array}{l}\text { Oे } \\
\text { Nે } \\
\text { N } \\
\text { Nి } \\
\text { ले }\end{array}$ & 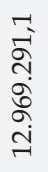 & 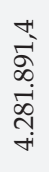 & 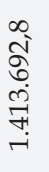 & 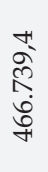 & 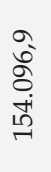 & 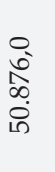 & 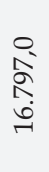 & 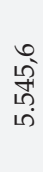 \\
\hline$a$ & 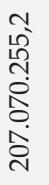 & 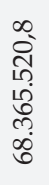 & 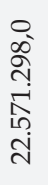 & 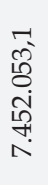 & $\begin{array}{l}m \\
\text { m } \\
\text { m. } \\
\text { o } \\
+1 \\
\text { i }\end{array}$ & 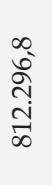 & 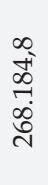 & $\begin{array}{l}\text { वे } \\
\text { ते } \\
\infty \\
\infty \\
\infty\end{array}$ & $\begin{array}{l}\text { ㅇ. } \\
\text { ते } \\
\text { ते } \\
\text { ते }\end{array}$ & $\begin{array}{l}\text { से } \\
\text { तु } \\
\sigma\end{array}$ & \\
\hline$\infty$ & 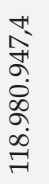 & 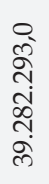 & 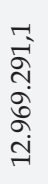 & 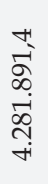 & 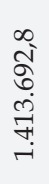 & 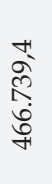 & 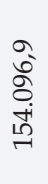 & 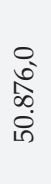 & 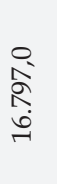 & & \\
\hline$\Lambda$ & 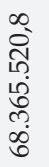 & 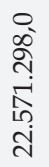 & 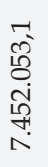 & 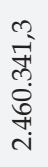 & 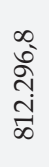 & 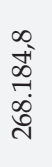 & $\begin{array}{l}\text { aे } \\
\text { Iै } \\
\infty \\
\infty \\
\infty\end{array}$ & $\begin{array}{l}\text { Oे } \\
\text { ते } \\
\text { ते } \\
\text { ते }\end{array}$ & & & \\
\hline 0 & 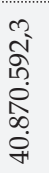 & 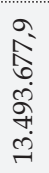 & 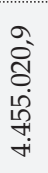 & 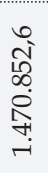 & 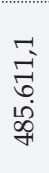 & 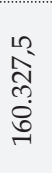 & $\begin{array}{l}\text { ते } \\
\text { ळे } \\
\hat{n} \\
\text { ñ }\end{array}$ & & & & \\
\hline in & 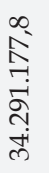 & $\begin{array}{l}\text { के } \\
\text { Fे } \\
\vec{\sim} \\
\text { యे } \\
\vec{F}\end{array}$ & 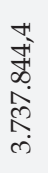 & 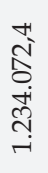 & 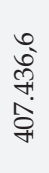 & 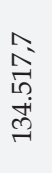 & & & & & \\
\hline tr & 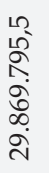 & 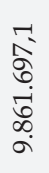 & 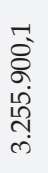 & 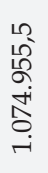 & 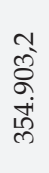 & & & & & & \\
\hline n & 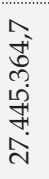 & 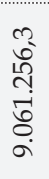 & 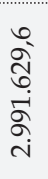 & 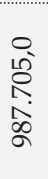 & & & & & & & \\
\hline$N$ & 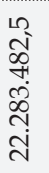 & 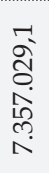 & 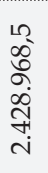 & & & & & & & & \\
\hline$r$ & 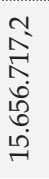 & 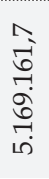 & & & & & & & & & \\
\hline
\end{tabular}


Valuación de un contrato de concesión petrolera: análisis del riesgo, simulación... https://doi.org/10.52292/j.eca.2021.2017

Tabla 27. Valor terminal $\mathrm{t}=10$ nodos $(\mathrm{i}=1, \mathrm{~J}=11)$

\begin{tabular}{c|c|c|c|c|c}
\hline Nodos & 11 & 10 & 9 & 8 & 7 \\
\hline $\mathbf{T = 1 0}(V(i, j)$ & 3.428 .709 .486 & 1.132 .009 .566 & $373.739 .934,5$ & $123.392 .542,6$ & $40.738 .808,35$ \\
\hdashline 6 & 5 & 4 & 3 & 2 & 1 \\
\hline $13.450 .168,63$ & $4.440 .656,062$ & $1.466 .109,965$ & $484.045,2399$ & $159.810,5189$ & $52.762,42768$ \\
\hline
\end{tabular}

Fuente: elaboración propia.

Recursivamente, se resuelve las opciones para el quinto y décimo años (ecuaciones 12 y 13), siendo el proceso recursivo de la rejilla el siguiente: , conforme se detalla en la tabla 28. 
Gastón Silverio Milanesi

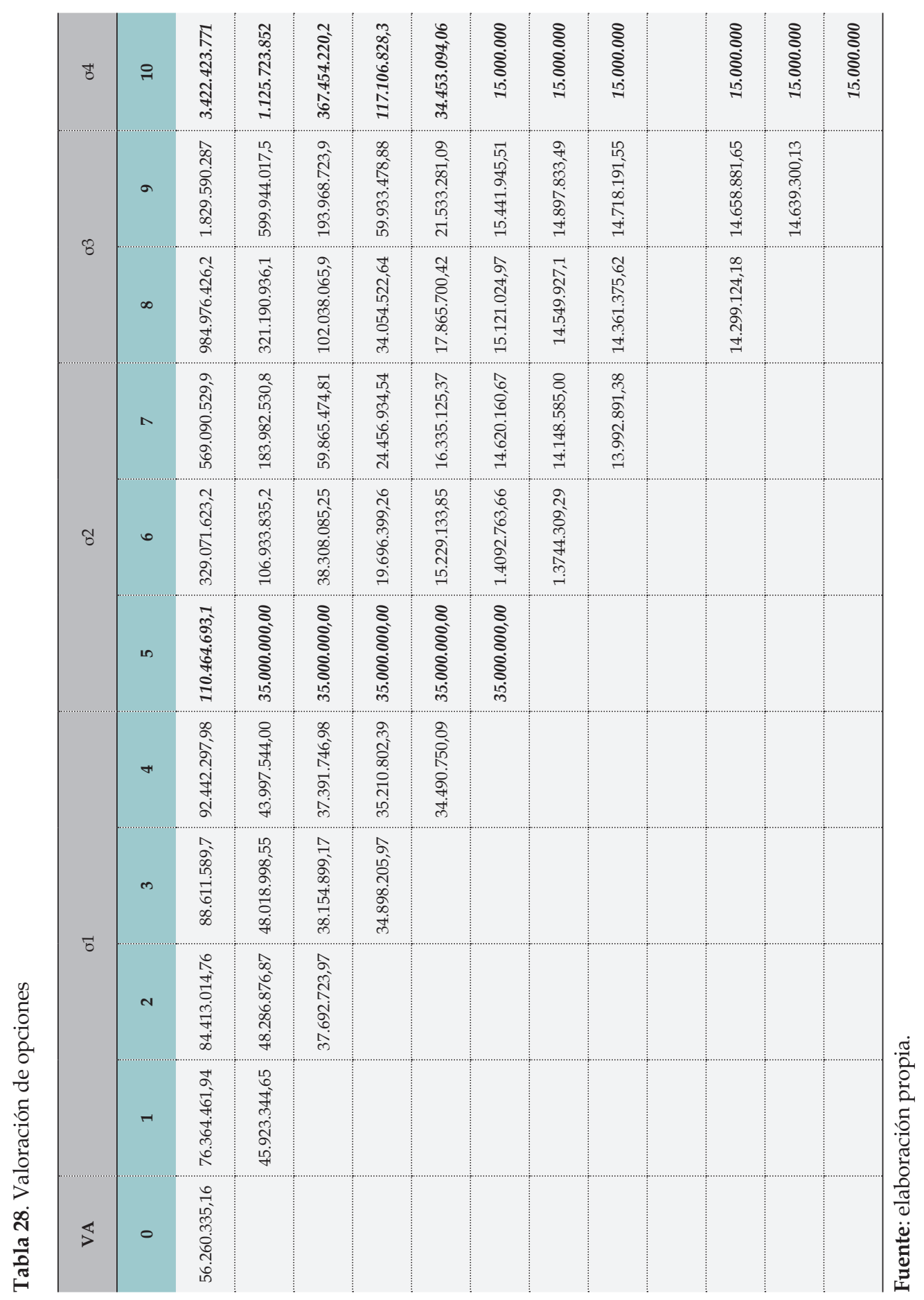


Valuación de un contrato de concesión petrolera: análisis del riesgo, simulación...

El valor actual del proyecto asciende a 56.260.335 USD, generando un VAN expandido de 5.411.756 USD, y un valor de las opciones reales contenidas en el proyecto de 179.051 USD.

Tabla 29. Ejercicio de opciones

\begin{tabular}{|c|c|c|c|}
\hline Nodo & $\mathrm{T}=5$ & Nodo & $\mathrm{T}=10$ \\
\hline 6 & continuar & 11 & continuar \\
\hline 5 & ceder & 10 & continuar \\
\hline 4 & ceder & 9 & continuar \\
\hline 3 & ceder & 8 & continuar \\
\hline 2 & ceder & 7 & continuar \\
\hline \multirow[t]{6}{*}{1} & ceder & 6 & ceder \\
\hline & & 5 & ceder \\
\hline & & 4 & ceder \\
\hline & & 3 & ceder \\
\hline & & 2 & ceder \\
\hline & & 1 & ceder \\
\hline
\end{tabular}

Fuente: elaboración propia.

La tabla 29 indica que en el momento $t=5$ solo conviene continuar solo en el nodo 6, es decir de ascenso total del crudo, mientras que el nodo $\mathrm{t}=10$ supone 5 nodos de continuación. El precio del crudo impacta negativamente en los proyectos de este tipo. No obstante, la información arrojada por el enfoque de opciones permite revisar el valor de las condiciones del contrato, en particular, los valores de renovación en $\mathrm{t}=5$ y 10 , respectivamente, con el objetivo de asegurar un $\mathrm{VAN}=0$. Este valor sirve para retribuir el costo del capital y factores de la producción. Se dispone como restricción que el valor del proyecto debe ser equivalente a 61.672.091 USD (pliego más inversiones) y se itera la rejilla binomial desarrollada en la tabla 29. Esta iteración se realiza por cláusula, suponiendo que el resto de las variables no cambia su comportamiento. Para ello se utiliza la función del aplicativo MS Excel, menú Datos, Análisis de Hipótesis, Buscar Objetivo, fijando como restricción la celda de valor expandido 
con valor 61.672.091 USD, cambiando la condición del contrato. Por ejemplo, el contrato debería permitir la cesión a otro operador en $\mathrm{t}=5,41.185 .575$ USD (6.000.000 USD adicionales al estipulado), o dar un subsidio en el caso de no presentarse el mejor escenario ( $\mathrm{t}=5$, nodo 6) de 30.676.418 USD.

Alternativamente, se puede plantear un subsidio de 5.411.756 USD, equivalente al VAN expandido negativo, disminuyendo el costo de la concesión a 56.260.355 USD. Este subsidio, en $\mathrm{t}=5$ equivale a 6.122.905 USD, aproximado al ajuste a la condición de cesión.

\section{Conclusiones}

El trabajo se planteó dos objetivos, uno descriptivo, donde se exponen los métodos usuales de valuación empleados por los practicantes y el empleo de la planilla de cálculo para estimar el riesgo del proyecto. El otro, presentar las ventajas de los modelos de opciones reales sobre los tradicionales, en particular para valorar la flexibilidad estratégica, en este caso con un modelo que permite abandonar el supuesto de volatilidad constante. El valor obtenido por el modelo de descuento de flujos de fondos no refleja la flexibilidad estratégica ni el comportamiento estocástico del precio del subyacente. Una representación más ajustada al comportamiento de las variables lo evidencia el modelo de opciones reales. A diferencia de un árbol de decisión tradicional prescinde de las probabilidades reales y tasas ajustadas por riesgos. En este caso, incorpora al valor las opciones contenidas en el pliego para los períodos de decisión. El modelo permite suponer niveles cambiantes de volatilidad, incluso estabilizar el comportamiento de las variables en el steady-state. Es un modelo que no pierde de vista el concepto de valor actual, pues la construcción de su valor es la suma del flujo de fondos libres en cada nodo y el valor actual de los flujos de fondos correspondientes a los futuros períodos. Es flexible, pues permite analizar y sensibilizar en un conjunto, las condiciones contractuales suponiendo como restricción un valor actual neto que retribuya a los factores de la producción y al cedente.

\section{Referencias}

Ahn, H., \& Wilmott, P. (2009). A note on hedging: restricted but optimal delta hedging, mean, variance, jumps, stochastic volatility, and costs. Wilmott Journal: The International Journal of Innovative Quantitative Finance Research, 1(3), 121-131. 
Valuación de un contrato de concesión petrolera: análisis del riesgo, simulación... https://doi.org/10.52292/j.eca.2021.2017

Amram, M., \& Kulatilaka, N. (1998). Real Options. Boston, Masachussets, Estados Unidos: Harvard Business School Prees.

Benninga, S. (2008). Financial Modeling (3 ed.). Cambridge, Massachussetts: The MIT Press.

Booth, L. (2002). Finding Value Where None Exists: Pitfalls in Using Adjusted Present Value. Journal of Applied Corporate Finance, 15(1), 8-17.

Booth, L. (2007). Capital Cash Flow, APV and Valuation. European Financial Management, 13(1), 29-48.

Brandao, L., Dyer, J., \& Hahn, W. (2005). Using binomial decision trees to solve real-option valuation problems. Decision Analysis, 2, 69-88.

Brandao, L., Dyer, J., \& Hahnn, W. (2012). Volatility estimation for stochastic project value models. European Journal of Operational Research, 220(3), 642-648.

Brous, P. (2011). Valuing an Early - Stage Biotechnology Investment as a Rainbow Option. Journal of Applied Corporate Finance, 23(2), 64-103.

Castro Monge, E. (2010). El estudio de casos como metodología de investigación y su importancia en la dirección y administración de empresas. Revista Nacional de Administración, 2(1), 31-54.

Chance, D. (2007). A Synthesis of Binomial Option Pricing Models for Lognormally Distributed Assets. SSRN http://ssrn.com/abstract=1523548, 1-25.

Copeland, T., Koller, K., \& Murrin, J. (2000). Valuation: Measuring and Managing the Value of Companies (3 ed.). New York: Wiley.

Copeland, T., Weston, F., \& Shastri, K. (2004). Financial Theory and Corporate Policy (4 ed.). Estados Unidos: Pearson Addison Wesley.

Copeland, T., \& Antikarov, V. (2003). Real Options: a practitioner's guide. New York: Cengage Learning.

Cox, J., Ross, S. y Rubinstein, M. (1979). Option Pricing: A Simplified Approach. Journal of Financial Economics, 7, 229-263.

Damodaran, A. (2006). Damodaran on Valuation (2 ed.). New York, United State: John Wiley \& Sons.

Damodaran, A. (2015). Country Risk: Determinants, Measures and Implications The 2015 edition. (SSRN, Ed.) Social Science Research Network (SSRN), Downloads/SSRN-id2630871.pdf, 1-97. https:/ / ssrn.com/abstract=4560293

Damodaran, A. (2020). Equity Risk Premiums: Determinants, Estimation and Implications - The 2020 Edition. Social Science Research Network (SSRN), Downloads/SSRN-id46391.pdf, 1-173. https:// ssrn.com/ abstract=3550293.

Dempsey, M. (2013). The Capital Asset Pricing Modelo (CAPM): The history of a failed revolutionary idea in Finance? Journal of Accounting, Finance and Business Studies, 49, 7-23. 
Dixit, A., \& Pindyck, R. (1994). Investment under Uncertainty. New Jersey: Pricenton University Press.

El Alabi, E., \& Milanesi, G. (2016). Evolución de las Funciones de Utilidad para la toma de decisiones. Escritos Contables y de Administración, 5(2), 43-79.

Esandi, J, Milanesi, G., \& Pesce, G. (2012). Análisis de escenario y opciones reales: un caso de aplicación para empresas de base tecnológica. XXXII Jornadas Sociedad Argentina de Docentes en Administración Financiera SADAF, 63-82.

Espert, J., \& Vignoli, G. (2018). Tipo de cambio real de largo plazo en Argentina: 1961-2017. Documento de trabajo: Universidad de CEMA(630).

Fernández, P. (2014). Valoración de Empresas y Sensatez (3 ed.). Barcelona: IESE Business School-Universidad de Navarra.

Haahtela, T. (2011a). Estimating Changing Volatility in Cash Flow Simulation Based Real Options Valuation with Regression Sum of Squared Error Method. SSRN: Social Science Research Network.

Haahtela, T. (2011b). Recombining trinomial tree for real option valuation with changing volatility. Annual Real Options Conference https://papers.ssrn.com/ sol3/papers.cfm?abstract_id=1932411, 1-25.

Machain, L. (2011). Simulación de Modelos Financieros. San Lorenzo: Machain.

Milanesi, G. (2011). Fuentes de Incertidumbre, Probabilidades Condicionales y la Opción de Abandono en Activos Reales. Escritos Contables y de Administración, 2(1), 43-71.

Milanesi, G. (2012). Opciones Reales: el Método Binomial, Asimetría y Curtosis en la Valoración de Empresas de Base Tecnológica. Revista Española de Capital de Riesgo (2), 41-55.

Milanesi, G. (2013a). El modelo binomial borroso y la valuación de opciones reales: el caso de valuación de un contrato de conseción para la explotación petrolera. Estocástica: Finanzas y Riesgo, 3(2), 95-118.

Milanesi, G. (2013b). Teoría de Opciones: Modelos específicos y aplicaciones para valorar estrategias, activos reales e instrumentos financieros. Bahía Blanca: EdiUNS-REUN.

Milanesi, G. (2014). Modelo Binomial para la Valoración de Empresas y los efectos de la Deuda: Escudo Fiscal y Liquidación de la Firma. Journal of Economics, Finance and Administrative Science, 19(36), 2-10.

Milanesi, G. (2016). Un modelo "naive" de opciones barreras para la predicción de fracasos financieros. Estocástica: Finanzas y Riesgo, 6(2), 159-186.

Milanesi, G. (2017). Valuación de empresas: enfoque integral para mercados emergentes e inflacionarios. Estudios Gerenciales, 33(145), 377-390. 
Milanesi, G. (2018). Un modelo de opciones reales fuzzy y funciones isoelásticas de utilidad para valorar I\&D en mercados incompletos. Estocástica: Administración y Riesgo, 8(2), 205-232.

Milanesi, G., Pesce, G., \& El Alabi, E. (2016). Firm valuation and default probability through exotic (barrier) options. European Accounting and Management Review, 2(2), 56-76.

Miller, K., \& Waller, G. (2003). Scenarios, Real Options and Integrated Risk Management. Journal of Long Range Planning, 36, 93-107.

Num, J. (2015). Real Options Analysis (Third Edition): Tools and Techniques for Valuing Strategic Investments and Decisions with Integrated Risk Management and Advanced Quantitative Decision Analytics (3 ed.). CreateSpace Independent Publishing Platform.

Pareja Vasseur, J., Prada Sánchez, M., \& Moreno Escobar, M. (2019). Volatilidad en Opciones Reales: Revisión literaria y un caso de aplicación al sector petrolero colombiano. Revista de Métodos Cuantitativos para la Economía y la Empresa (27), 136-155.

Pratt, S., \& Grabowski, R. (2008). Cost Of Capital: Applications and Examples (3 ed.). New Jersey: John Wiley \& Sons.

Smit, H. \& Trigeorgis, L. (2004). Strategic Investment: Real Options and Games. New Jersey, Estados Unidos: Princeton University Press.

Smith, J. (2005). Alternative Approach for Solving Real Options Problems. Decision Analysis (2), 89-102.

Smith, J., \& Nau, R. (1995). Valuing Risky Projects: Option Pricing Theory and Decision Anaysis. Management Science (5), 795-816.

Trigeorgis, L. (1995). Real Options in Capital Investment: Models, Strategies and Applications. London, United Kindgon: Praeger.

Trigeorgis, L. (1997). Real Options: Managerial Flexibility and Strategy in Resource Allocations (2 ed.). Cambridge: MIT Press.

Van der Hoek, J., \& Elliot, R. (2006). Binomial models in Finance. New York, United State: Springer Science.

Whaley, R. (2006). Derivatives, Markets, Valuation and Risk Management. New Jersey: John Wiley \& Sons. 


\section{Apéndice}

\section{Proyección del flujo de fondos determinístico en base a estimaciones puntuales}

Tabla A.1. Flujo de fondos determinístico

\begin{tabular}{|c|c|c|c|c|c|c|c|c|c|c|c|c|}
\hline \multirow{2}{*}{$\begin{array}{l}\text { Proyectado } \\
\text { determinístico }\end{array}$} & \multicolumn{11}{|c|}{ Horizonte de Proyección } & \multirow[b]{2}{*}{ Total } \\
\hline & 0 & 1 & 2 & 3 & 4 & 5 & 6 & 7 & 8 & 9 & 10 & \\
\hline $\begin{array}{l}\text { Producción m3 } \\
\text { anual por pozo }\end{array}$ & & 42.000 & 33.600 & 23400 & 14.400 & 9.600 & 6.600 & 6.600 & 6.600 & 6.600 & 6.600 & 156.000 \\
\hline $\begin{array}{l}\text { Producción bbl } \\
\text { anual por pozo }\end{array}$ & & 264.180 & 211.344 & 147.186 & 90.576 & 60.384 & 41.514 & 41.514 & 41.514 & 41.514 & 41.514 & 981.240 \\
\hline $\begin{array}{l}\text { Producción m3 } \\
\text { anual total }\end{array}$ & & 168.000 & 134.400 & 93.600 & 57.600 & 38.400 & 26.400 & 26.400 & 26.400 & 26.400 & 26.400 & 624.000 \\
\hline $\begin{array}{l}\text { Producción bbl } \\
\text { anual total }\end{array}$ & & 1.056 .720 & 845.376 & 588.744 & 362.304 & 241.536 & 166.056 & 166.056 & 166.056 & 166.056 & 166.056 & 3.924 .960 \\
\hline $\begin{array}{l}\text { Inflación EE.UU } \\
\text { Proyectada }\end{array}$ & & $2,73 \%$ & $2,27 \%$ & $2,23 \%$ & $2,24 \%$ & $2,23 \%$ & $2,20 \%$ & $2,00 \%$ & $2,00 \%$ & $2,00 \%$ & $2,00 \%$ & \\
\hline $\begin{array}{l}\text { Inflación Argentina } \\
\text { Proyectada }\end{array}$ & & $30,33 \%$ & $24,22 \%$ & $20,89 \%$ & $18,55 \%$ & $16,70 \%$ & $15,16 \%$ & $13,66 \%$ & $12,55 \%$ & $11,57 \%$ & $10,69 \%$ & \\
\hline Tipo de cambio & 79,3 & 100,6 & 122,1 & 144,4 & 167,5 & 191,2 & 215,4 & 240,1 & 264,9 & 289,8 & 314,5 & \\
\hline $\begin{array}{l}\text { Precio brent pro- } \\
\text { medio } 5\end{array}$ & & 58,4 & 58,4 & 58,4 & 58,4 & 58,4 & 58,4 & 58,4 & 58,4 & 58,4 & 58,4 & \\
\hline $\begin{array}{l}\text { Precio brent prome- } \\
\text { dio } 10\end{array}$ & & 79,9 & 79,9 & 79,9 & 79,9 & 79,9 & 79,9 & 79,9 & 79,9 & 79,9 & 79,9 & \\
\hline Costos desarrollo & & 10,5 & 10,5 & 10,5 & 10,5 & 10,5 & 10,5 & 10,5 & 10,5 & 10,5 & 10,5 & \\
\hline $\begin{array}{l}\text { Costo producción } \\
\text { (lifting cost) }\end{array}$ & & 12,5 & 12,5 & 12,5 & 12,5 & 12,5 & 12,5 & 12,5 & 12,5 & 12,5 & 12,5 & \\
\hline Regalias & & 10,0 & 10,0 & 10,0 & 10,0 & 10,0 & 10,0 & 10,0 & 10,0 & 10,0 & 10,0 & \\
\hline Break even & & 33,0 & 33,0 & 33,0 & 33,0 & 33,0 & 33,0 & 33,0 & 33,0 & 33,0 & 33,0 & \\
\hline $\begin{array}{l}\text { EBIT bbl brent } \\
\text { promedio } 5\end{array}$ & & 25,4 & 25,4 & 25,4 & 25,4 & 25,4 & 25,4 & 25,4 & 25,4 & 25,4 & 25,4 & \\
\hline $\begin{array}{l}\text { EBIT bbl brent } \\
\text { promedio } 10\end{array}$ & & 46,9 & 46,9 & 46,9 & 46,9 & 46,9 & 46,9 & 46,9 & 46,9 & 46,9 & 46,9 & \\
\hline $\begin{array}{l}\text { Ingresos totales } \\
\text { brent promedio } 5\end{array}$ & & $61.736 .007,7$ & $49.388 .806,1$ & $34.395 .775,7$ & $21.166 .631,2$ & $14.111 .087,5$ & $9.701 .372,6$ & $9.701 .372,6$ & $9.701 .372,6$ & $9.701 .372,6$ & $9.701 .372,6$ & $229.305 .171,3$ \\
\hline $\begin{array}{l}\text { Ingresos totales } \\
\text { brent promedio } 10\end{array}$ & & $84.449 .066,4$ & $67.559 .253,1$ & $47.050 .194,1$ & $28.953 .965,6$ & $19.302 .643,7$ & $13.270 .567,6$ & $13.270 .567,6$ & $13.270 .567,6$ & $13.270 .567,6$ & $13.270 .567,6$ & $313.667 .960,9$ \\
\hline $\begin{array}{l}\text { Costos totales } \\
\text { desarrollo }\end{array}$ & & $11.095 .560,0$ & $8.876 .448,0$ & $6.181 .812,0$ & $3.804 .192,0$ & $2.536 .128,0$ & $1.743 .588,0$ & $1.743 .588,0$ & $1.743 .588,0$ & $1.743 .588,0$ & $1.743 .588,0$ & $41.212 .080,0$ \\
\hline $\begin{array}{l}\text { Costo totales pro- } \\
\text { ducción (lifting cost) }\end{array}$ & & $13.219 .567,2$ & $10.575 .653,8$ & $7.365 .187,4$ & $4.532 .423,0$ & $3.021 .615,4$ & $2.077 .360,6$ & $2.077 .360,6$ & $2.077 .360,6$ & $2.077 .360,6$ & $2.077 .360,6$ & $49.101 .249,6$ \\
\hline Regalias totals & & $10.567 .200,0$ & $8.453 .760,0$ & $5.887 .440,0$ & $3.623 .040,0$ & $2.415 .360,0$ & $1.660 .560,0$ & $1.660 .560,0$ & $1.660 .560,0$ & $1.660 .560,0$ & $1.660 .560,0$ & $39.249 .600,0$ \\
\hline Break even total & & $34.882 .327,2$ & $27.905 .861,8$ & $19.434 .439,4$ & $11.959 .655,0$ & $7.973 .103,4$ & $5.481 .508,6$ & $5.481 .508,6$ & $5.481 .508,6$ & $5.481 .508,6$ & $5.481 .508,6$ & $129.562 .929,6$ \\
\hline $\begin{array}{l}\text { EBIT brent precio } \\
\text { promedio } 5\end{array}$ & & $26.853 .680,5$ & $21.482 .944,4$ & $14.961 .336,3$ & $9.206 .976,2$ & $6.137 .984,1$ & $4.219 .864,1$ & $4.219 .864,1$ & $4.219 .864,1$ & $4.219 .864,1$ & $4.219 .864,1$ & $99.742 .241,7$ \\
\hline $\begin{array}{l}\text { EBIT brent prome- } \\
\text { dio } 10\end{array}$ & & $49.566 .739,2$ & $39.653 .391,4$ & 127.615.754,7 & $16.994 .310,6$ & $11.329 .540,4$ & $7.789 .059,0$ & $7.789 .059,0$ & $7.789 .059,0$ & $7.789 .059,0$ & $7.789 .059,0$ & $184.105 .031,3$ \\
\hline $\begin{array}{l}\text { Tax/EBIT brent } \\
\text { promedio } 5\end{array}$ & & $8.056 .104,1$ & $6.444 .883,3$ & $4.488 .400,9$ & $2.762 .092,8$ & $1.841 .395,2$ & $1.265 .959,2$ & $1.265 .959,2$ & $1.265 .959,2$ & $1.265 .959,2$ & $1.265 .959,2$ & $29.922 .672,5$ \\
\hline $\begin{array}{l}\text { Tax/EBIT brent } \\
\text { promedio } 10\end{array}$ & & $14.870 .021,8$ & $11.896 .017,4$ & $8.284 .726,4$ & $5.098 .293,2$ & $3.398 .862,1$ & $2.336 .717,7$ & $2.336 .717,7$ & $2.336 .717,7$ & $2.336 .717,7$ & $2.336 .717,7$ & $55.231 .509,4$ \\
\hline $\begin{array}{l}\text { FFO brent prome- } \\
\text { dio } 5\end{array}$ & & $18.797 .576,3$ & $15.038 .061,1$ & $10.472 .935,4$ & $6.444 .883,3$ & $4.296 .588,9$ & $2.953 .904,9$ & $2.953 .904,9$ & $2.953 .904,9$ & $2.953 .904,9$ & $2.953 .904,9$ & $69.819 .569,2$ \\
\hline
\end{tabular}


Valuación de un contrato de concesión petrolera: análisis del riesgo, simulación... https://doi.org/10.52292/j.eca.2021.2017

\begin{tabular}{|c|c|c|c|c|c|c|c|c|c|c|c|c|}
\hline \multirow{2}{*}{$\begin{array}{c}\text { Proyectado } \\
\text { determinístico }\end{array}$} & \multicolumn{11}{|c|}{ Horizonte de Proyección } & \multirow[b]{2}{*}{ Total } \\
\hline & 0 & 1 & 2 & 3 & 4 & 5 & 6 & 7 & 8 & 9 & 10 & \\
\hline $\begin{array}{l}\text { FFO brent prome- } \\
\text { dio } 10\end{array}$ & & $34.696 .717,4$ & $27.757 .374,0$ & $19.331 .028,3$ & $11.896 .017,4$ & $7.930 .678,3$ & $5.452 .341,3$ & $5.452 .341,3$ & $5.452 .341,3$ & $5.452 .341,3$ & $5.452 .341,3$ & \multirow[t]{3}{*}{$128.873 .521,9$} \\
\hline $\begin{array}{l}\text { Inversión capital de } \\
\text { trabajo }\end{array}$ & $1.672 .091,5$ & $1.672 .091,5$ & $1.337 .673,2$ & $931.593,8$ & $573.288,5$ & $382.192,3$ & $262.757,2$ & $262.757,2$ & $26.2757,2$ & $262.757,2$ & $262.757,2$ & \\
\hline $\begin{array}{l}\text { Inversión incremen- } \\
\text { tal NWC }\end{array}$ & & 0,0 & $-334.418,3$ & $-406.079,4$ & $-358.305,3$ & $-191.096,2$ & $-119.435,1$ & 0,0 & 0,0 & 0,0 & 0,0 & \\
\hline NWC acumulado & $1.672 .091,5$ & $1.672 .091,5$ & $1.337 .673,2$ & $931.593,8$ & $573.288,5$ & $382.192,3$ & $262.757,2$ & $262.757,2$ & $262.757,2$ & $262.757,2$ & $262.757,2$ & 262757,2 \\
\hline Inversión inicial & $60.000 .000,0$ & & & & & & & & & & & $60.000 .000,0$ \\
\hline $\begin{array}{l}\text { FFL brent prome- } \\
\text { dio } 5\end{array}$ & $-61.672 .091,5$ & $18.797 .576,3$ & $15.372 .479,4$ & $10.879 .014,7$ & $6.803 .188,6$ & $4.487 .685,0$ & $3.073 .340,0$ & $2953.904,9$ & $2.953 .904,9$ & $2.953 .904,9$ & $2.953 .904,9$ & $9.556 .812,0$ \\
\hline $\begin{array}{l}\text { FFL brent prome- } \\
\text { dio } 10\end{array}$ & $-61.672 .091,5$ & $34.696 .717,4$ & $28.091 .792,3$ & $19.737 .107,7$ & $12.254 .322,7$ & $8.121 .774,4$ & $5.571 .776,4$ & $5.452 .341,3$ & $5.452 .341,3$ & $5.452 .341,3$ & $5.452 .341,3$ & $68.610 .764,7$ \\
\hline CC (ku) & & $26,82 \%$ & $26,36 \%$ & $26,32 \%$ & $26,33 \%$ & $26,32 \%$ & $26,29 \%$ & $26,09 \%$ & $26,82 \%$ & $26,82 \%$ & $26,82 \%$ & $26,82 \%$ \\
\hline Descuento & & 1,268164 & 1,602406376 & 2,024102048 & 2,55697525 & 3,229879084 & 4,07889802 & 5,142935673 & 6,522085875 & 8,271074512 & 10,48907894 & 13,3018723 \\
\hline $\begin{array}{l}\text { VA FFL brent } \\
\text { promedio } 5\end{array}$ & $36.260 .341,6$ & $14.822 .669,9$ & $9.593 .371,3$ & $5.374 .736,3$ & $2660.639,2$ & $1.389 .428,2$ & $753.473,1$ & $574.361,6$ & $452.908,0$ & $357.136,8$ & $281.617,2$ & \\
\hline $\begin{array}{l}\text { VA FFL brent } \\
\text { promedio } 10\end{array}$ & $66.390 .093,6$ & $27.359 .803,2$ & $17.531 .003,8$ & $9.751 .043,8$ & $4.792 .507,4$ & $2.514 .575,4$ & $1.366 .000,4$ & $1.060 .161,3$ & $835.981,2$ & $659.205,9$ & $519.811,3$ & \\
\hline
\end{tabular}

Fuente: elaboración propia.

(C) 2021 por los autores; licencia otorgada a la revista Escritos Contables y de Administración. Este artículo es de acceso abierto y distribuido bajo los términos y condiciones de una licencia Atribución-No Comercial 4.0 Internacional (CC BY-NC 4.0) de Creative Commons. Para ver una copia de esta licencia, visite https://creativecommons.org/licenses/by-nc/4.0/ 